\title{
Energetically stable discretizations for charge transport and electrokinetic models
}

\author{
Chun Liu ${ }^{1}$ \\ Department of Mathematics, The Pennsylvania State University, University Park, \\ Pennsylvania 16802.Email: cxl41@psu.edu. \\ Jinchao $\mathrm{Xu}^{2}$ \\ Department of Mathematics, The Pennsylvania State University, University Park, \\ Pennsylvania 16802. Email: xu@math.psu.edu. \\ Maximilian S. Metti*,3 \\ Department of Mathematics, The Pennsylvania State University, University Park, \\ Pennsylvania 16802. Email: msm37@psu.edu.
}

\begin{abstract}
A finite element discretization using a method of lines approached is proposed for approximately solving the Poisson-Nernst-Planck (PNP) equations. This discretization scheme enforces positivity of the computed solutions, corresponding to particle density functions, and a discrete energy estimate is established that takes the same form as the energy law for the continuous PNP system. This energy estimate is extended to finite element solutions to an electrokinetic model, which couples the PNP system with the incompressible Navier-Stokes equations. Numerical experiments are conducted to validate convergence of the computed solution and verify the discrete energy estimate.

Key words: Finite elements, Poisson-Nernst-Planck, stability analysis, energy estimate

\footnotetext{
* Corresponding Author

${ }^{1}$ The work of this author was partially supported by NSF grants DMS-1412005 and DMS1216938.

${ }^{2}$ The work of this author was supported by the DOE Grant DE-SC0009249 as part of the Collaboratory on Mathematics for Mesoscopic Modeling of Materials.

${ }^{3}$ The work of this author was supported by the DOE Grant DE-SC0009249 as part of the Collaboratory on Mathematics for Mesoscopic Modeling of Materials.
}

Preprint submitted to Journal of Computational Physics

October 6, 2015

(c) 2015. This manuscript version is made available under the Elsevier user license http://www.elsevier.com/open-access/userlicense/1.0/ 


\section{Introduction and Background}

Charge transport refers to a physical process where charge carriers interact with an electric field, so that their transport not only affects the configuration of other charge carriers, but also has a global influence on the electric field. As a result, these systems require strong coupling between the charge carriers and the electric field. To be clear, a charge carrier in this setting is a general concept of some entity that has a density and an electrical charge, which includes physical particles, such as an ion or electron, as well as massless quasi-particles, like the conduction band "electrons" and valence band holes studied in semiconductor literature.

Charge transport systems have been observed throughout the history of science; they are, naturally, the foundation of electrical engineering and electrokinetics $[9,20,24,27,28,31]$; and, they are commonplace in physical systems and biological systems $[9,10,14,26,42,45]$. From a mathematical perspective, charge transport fits into a setting of generalized diffusion, where the charge carriers have nonlocal influence upon each other through Coulomb interactions, provided by the electric potential. This nonlocal diffusion fits into an energetic variational framework, which is a useful tool for establishing self-consistent model equations and various qualitative properties of the system, such as an energy law $[10,18,15]$.

Our model for charge transport is described by the Poisson-Nernst-Planck (PNP) system of differential equations, which models an electrostatic system (where the effects of magnetic forces are ignored), and is derived from three key concepts. The first is Gauss' law, from the Maxwell equations [29], which states that the electrostatic potential is distributed according to the total charge density in the system via Poisson's equation. The second ingredient is that the fluxes of the charge carriers are driven exclusively by processes of diffusion and electric drift. This definition for the flux traces back to Nernst [32] and Planck [33] and is accurate for describing systems where charge carriers can be modeled as point charges. Finally, the conservation of mass is used to relate the evolution of the charge carrier densities to the Nernst-Planck flux.

An important remark about the PNP system is that it has many variants by simply altering the definition of the charge carrier flux. For example, some practical modifications take into account size effects of the ionic particles [14, 17] or relative drag when there is ion crowding [10, 15]. Another important extension to the Nernst-Planck flux is to include an advection term that is coupled to the incompressible Navier-Stokes (NS) equations with an electricbody force, which models charge carriers that are suspended in a charged fluid. The PNP-NS system is a basic model in the study of electrokinetics [24] and is important to engineering lab-on-chip devices, for example, where a fluid carries charged particles or the charged particles are used to control fluid flow. For the

purposes of this paper, only the Nernst-Planck flux or the Nernst-Planck flux coupled to the NS equations are considered.

The PNP equations serve as the basis for modeling many devices, such as batteries [30,43], semiconductor devices [3, 6, 9, 19, 20, 27, 28, 31, 41], fluidic 
micro/nano-channels and mixers [11, 21, 24, 40], and biological ion channels [10, $13,26,42,45]$. As a system of coupled nonlinear partial differential equations, the PNP equations lead to a rich source of problems for pde analysis, where the system and its modifications are studied to improve understanding of the existence, uniqueness, and stability of a solution $[4,5,19,36]$.

Due to the wide variety of devices modeled by the PNP equations, computer simulation for this system of differential equations is of remarkable interest. This has led to a great deal of literature focusing on numerical solvers for the PNP systems $[3,6,16,20,26,30,34,35,37,42,43,45]$. Providing a comprehensive numerical analysis would require an energy estimate to establish the stability of the discretization, some notion of convergence of the computed solution to the true solution, and well-posedness of the discrete problem.

It is worth noting that there are many ways in which one can approach the simulation of the PNP system. To start, the weak form of this system is not unique; Prohl and Schmuck carried out an analysis for the PNP system [34] and the PNP system coupled with the incompressible Navier-Stokes equation [35] that uses the $\mathcal{L}_{2}$ inner-product to define the weak from. Their work is significant as they define a numerical scheme for which they establish convergence and an energy estimate; however, due to their definition of the weak form using the $\mathcal{L}_{2}$ inner-product, the energy estimate is an imitation of the physically relevant energy norm, which is defined using the $\mathcal{L}_{1} \log \mathcal{L}_{1}$-norm. As a result, the self-consistent energy norm cannot be evaluated exactly in using an $\mathcal{L}_{2^{-}}$ discretization, and interpolation must be used to approximate the energy law that governs the system.

In this work, a novel finite element discretization is used that employs a logarithmic transformation of the charge carrier densities that naturally yields several favorable properties, such as automatic positivity of the solution densities and the energetic stability of the numerical solution for both the drift-diffusion model and the electrokinetic model. It is important to note that this stability is always true for the nonlinear finite element solution, regardless of the mesh size. In $\S 2$, we define the PNP equations, introduce the energy law corresponding to the PNP system, propose a finite element discretization, and prove an energy estimate for the fully discrete solution to the PNP system. In $\S 3$, we provide a similar analysis for the PNP system coupled with an incompressible fluid, where the divergence-free property of the fluid plays an essential role in establishing stability; consequently, this section also contains a discussion of a discontinuous Galerkin discretization for the incompressible Navier-Stokes system, which is known to preserve this divergence-free property. In $\S 4$, some numerical experiments are carried out to validate the convergence properties of the numerical solver and the discrete energy estimate. Some closing remarks are given in $\S 5$.

\section{The Poisson-Nernst-Planck equations and its discretization}

The PNP system models the interaction of $N \geq 2$ charge carriers through an electrostatic field. Denote the charge carrier density of the $i^{\text {th }}$ species by $\rho_{i}>0$ and the electrostatic potential by $\phi$. Let $\Omega \subset \mathbb{R}^{d}$ for $d=1,2$, or 3 be a simply 
connected polyhedral domain, and $T$ be a positive and finite real number. Then, the PNP system is described by the initial-boundary value problem:

$$
\begin{aligned}
-\nabla \cdot(\epsilon \nabla \phi) & =e_{c} \sum_{i=1}^{N} q_{i} \rho_{i}, & & \\
\frac{\partial \rho_{i}}{\partial t} & =-\nabla \cdot \vec{J}_{i}, & & i=1, \ldots, N, \\
\vec{J}_{i} & =-D_{i} \nabla \rho_{i}-q_{i} \mu_{i} \rho_{i} \nabla \phi, & & i=1, \ldots, N,
\end{aligned}
$$

in $\Omega \times(0, T]$, where

$$
\rho_{i}(x, 0)=\rho_{i, 0}(x), \quad \quad \text { for } x \in \Omega, i=1, \ldots, N,
$$

and

$$
-\nabla \cdot(\epsilon \nabla \phi(x, 0))=e_{c} \sum_{i=1}^{N} q_{i} \rho_{i, 0}(x), \quad \text { for } x \in \Omega .
$$

The electric permittivity, $\epsilon=\epsilon_{r} \epsilon_{0}>0$, is the product of the vacuum permittivity constant, $\epsilon_{0}$, and the material dependent relative permittivity, $\epsilon_{r}$, which may be discontinuous in general. The electric permittivity measures the strength of the long-range (nonlocal) interactions of the charge carriers. The elementary charge constant is approximately given by $e_{c}=1.6 \times 10^{-19}$ coulombs. The charge carrier flux for the $i^{\text {th }}$ species is denoted by $\vec{J}_{i}$ and is defined in (3) by a model proposed by Nernst and Planck [32,33], where $D_{i}>0$ is the diffusivity, $q_{i}$ the valency, and $\mu_{i}$ the mobility of the $i^{\text {th }}$ charge carrier. This model is reasonable when the charge carriers are sufficiently small (with respect to the length scale of the domain) to be accurately modeled as point charges.

The Einstein relation is assumed to hold so that

$$
\mu_{i}=e_{c} D_{i} / \kappa_{B} T^{\circ},
$$

which implies that equilibrium distribution of the charge carriers should follow a Boltzmann distribution. Here, $\kappa_{B}$ is the Boltzmann constant and $T^{\circ}$ is the temperature, which is considered to be fixed for the purposes of this paper. For simplicity, the equations are non-dimensionalized to give $e_{c}=1$ and $\kappa_{B} T^{\circ}=1$, so that $\mu_{i}=D_{i}$.

\subsection{Boundary conditions}

The boundary conditions are a critical component of the PNP model and determine important qualitative behavior of the solution. A detailed account of stability and existence for steady-state continuous and finite element solutions has reported in $[19,20]$. For the time-dependent problem, existence and stability for the continuous case has been established $[4,5]$. This work is concerned with establishing the stability of finite element solutions for the time dependent 
PNP equations, as in the work of Prohl and Schmuck [34, 35]; though, the discretization proposed in this work is markedly different from their presentation. Homogeneous no-flux conditions are considered for each charged species,

$$
D_{i}\left(\nabla \rho_{i}+q_{i} \rho_{i} \nabla \phi\right) \cdot \vec{n}=\overrightarrow{J_{i}} \cdot \vec{n}=0, \quad \text { on } \partial \Omega .
$$

For the Poisson equation, write a disjoint partition of the boundary: $\partial \Omega=$ $\Gamma_{D} \cup \Gamma_{N} \cup \Gamma_{R}$ with

$$
\begin{aligned}
\phi & =\delta V & & \text { on } \Gamma_{D}, \\
\epsilon \nabla \phi \cdot \vec{n} & =S & & \text { on } \Gamma_{N}, \\
\epsilon \nabla \phi \cdot \vec{n}+\kappa \phi & =C & & \text { on } \Gamma_{R},
\end{aligned}
$$

where $\delta V, S$ and $C$ are given functions that do not depend on time. Dirichlet boundary conditions model an applied voltage; Neumann conditions model surface charges; and, Robin conditions model capacitors at the boundary. Without loss of generality, it is assumed that $\int_{\Gamma_{D}} \delta V d s=0$, so that $\delta V \equiv 0$ if the Dirichlet boundary condition is constant. The capacitance is required to be positive on $\Gamma_{R}, \kappa \geq \bar{\kappa}>0$, though one may take $\Gamma_{R}=\emptyset$ if no capacitor boundary is to be modeled. Any combination of Dirichlet, Neumann, and Robin boundary conditions can be applied to $\phi$ for the purposes of this paper, though the case of pure Neumann boundary conditions requires an additional constraint, which is taken to be $\int_{\Omega} \phi(x, t) d x=0$ for $0 \leq t \leq T$, so that $\phi$ is uniquely defined.

\subsection{Computational difficulties of the PNP system}

The PNP equations present several difficulties when computing approximate solutions. Firstly, it is a strongly coupled system of $N+1$ nonlinear equations, so that computational efficiency plays a critical role in applications of a numerical solver. Furthermore, to resolve the nonlinearities of the system, an iterative linearization technique must be used, such as a Newton-Raphson method or fixed point iteration. While fixed point iteration serves as a helpful tool in the analysis of the PNP system, it is difficult to establish the rate of convergence to the nonlinear solution. Secondly, the Nernst-Planck fluxes given in (3) are often convection-dominated, which leads to several analytical and numerical difficulties, such as the positivity of the ion concentrations, $\rho_{i}>0$, or numerically induced oscillations in the computed solution, if not properly addressed. There are several ways to overcome these issues: one reasonable approach is to introduce some sort of upwinding scheme, such as the Scharfetter-Gummel scheme or box method [2,3,38], or the edge-averaged finite element method $[23,44]$. Another option is to introduce a nonlinear change of variables, such as the Slotboom variables [30, 41, 42] or the quasi-Fermi variables [2, 3, 19, 20], which symmetrize the Nernst-Planck flux. While the Slotboom change of variables has been used for several numerical solvers, it is known that this change of variables suffers from stability issues [26, 37].

In this work, a novel change of variables converts the convection-dominated Nernst-Planck flux into a nonlinearly diffusive flux, similar to the quasi-Fermi 
variables. As a matter of fact, this change of variables is directly related to the quasi-Fermi variables, though the quasi-Fermi variables introduce an additional nonlinear coupling between the charge carriers and the electrostatic potential in the time derivative term of (2), which is avoided here. The motivation for the proposed change of variables is inspired by energy law of the PNP system, which is discussed below.

\subsection{Energy of the PNP system}

Since the PNP system is assumed to satisfy the no-flux boundary condition for the charge carrier concentrations (4), it is known that the charge carrier concentrations satisfy the conservation of mass,

$$
\int_{\Omega} \rho_{i}(x, t) d x=\int_{\Omega} \rho_{i, 0}(x) d x, \quad \text { for } 0 \leq t \leq T .
$$

Furthermore, assume the Dirichlet boundary condition is homogeneous, then the stability of the solution to the PNP system is known $[4,5]$ to be given by the familiar energy law

$$
\begin{aligned}
\frac{d}{d t}\left\{\int_{\Omega} \sum_{i=1}^{N} \rho_{i}\left(\log \rho_{i}-1\right)+\frac{\epsilon}{2}|\nabla \phi|^{2}\right. & \left.d x+\int_{\Gamma_{R}} \frac{\kappa}{2}|\phi|^{2} d s\right\} \\
& =-\int_{\Omega} \sum_{i=1}^{N} D_{i} \rho_{i}\left|\nabla\left(\log \rho_{i}+q_{i} \phi\right)\right|^{2} d x
\end{aligned}
$$

where the functional,

$$
\int_{\Omega} \sum_{i=1}^{N} \rho_{i}\left(\log \rho_{i}-1\right)+\frac{\epsilon}{2}|\nabla \phi|^{2} d x+\int_{\Gamma_{R}} \frac{\kappa}{2}|\phi|^{2} d s,
$$

is the energy and

$$
\int_{\Omega} \sum_{i=1}^{N} D_{i} \rho_{i}\left|\nabla\left(\log \rho_{i}+q_{i} \phi\right)\right|^{2} d x \geq 0,
$$

is the rate of dissipation. The physical relevance of the no-flux boundary conditions on the charge carrier concentration and the no-voltage boundary conditions on $\phi$ stem from the notion that the PNP system is energetically closed; that is, there is no direct input or output of energy at the boundary.

In many applications of the PNP system, however, the case of inhomogeneous Dirichlet boundary conditions is critically important. For example, simulations of semiconductor devices, protein nano-channels, and electrokinetic devices are designed to observe qualitative behavior of the solution when a nonzero voltage is applied across the device. Therefore, it is important that the stability of such 
systems is established. Assume that the Dirichlet boundary condition does not depend on time and define $\phi_{D}$ to be the function that satisfies

$$
\begin{aligned}
& -\nabla \cdot\left(\epsilon \nabla \phi_{D}\right)=0 \quad \text { in } \Omega, \quad \phi_{D}=\delta V \quad \text { on } \Gamma_{D} \\
& \epsilon \nabla \phi_{D} \cdot \vec{n}+\kappa \phi_{D}=0 \quad \text { on } \Gamma_{R}, \quad \nabla \phi_{D} \cdot \vec{n}=0 \quad \text { on } \Gamma_{N} .
\end{aligned}
$$

Then, the corresponding energy law is given by

$$
\begin{array}{r}
\frac{d}{d t}\left\{\int_{\Omega} \sum_{i=1}^{N} \rho_{i}\left(\log \rho_{i}-1\right)+\frac{\epsilon}{2}|\nabla \phi|^{2}\right. \\
\left.+\sum_{i=1}^{N} q_{i} \rho_{i} \phi_{D} d x+\int_{\Gamma_{R}} \frac{\kappa}{2}|\phi|^{2} d s\right\} \\
=-\int_{\Omega} \sum_{i=1}^{N} D_{i} \rho_{i}\left|\nabla\left(\log \rho_{i}+q_{i} \phi\right)\right|^{2} d x,
\end{array}
$$

where the energy is bounded below by the conservation of mass (6) and a maximum principle for $\phi_{D}$ :

$$
\int_{\Omega} \sum_{i=1}^{N} q_{i} \rho_{i}(t) \phi_{D} d x \geq-\max _{x \in \Gamma_{D}}|\delta V(x)| \sum_{i=1}^{N}\left|q_{i}\right| \int_{\Omega} \rho_{i, 0}(x) d x .
$$

Aside from the additional term arising from inhomogeneous boundary conditions, the energy associated with this system takes an unusual form compared to those typically encountered in finite element analysis due to the presence of the logarithm. Even so, these identities establish the stability of the system and prescribe the rate of energy dissipation. To define the solution space of the PNP system, some standard spaces must be defined. For $1 \leq p<\infty$, let $\mathcal{L}_{p}(\Omega)=$ $\left\{f: \Omega \rightarrow \mathbb{R} \mid\left[\int_{\Omega}|f(x)|^{p} d x\right]^{1 / p}<\infty\right\}$, and $\mathcal{H}^{1}(\Omega)=\left\{f \in \mathcal{L}_{2}(\Omega) \mid \partial f / \partial x_{i} \in\right.$ $\mathcal{L}_{2}(\Omega)$ for $\left.i=1, \ldots, d\right\}$. These spaces are equipped with the usual norms: for $1 \leq p<\infty$

$$
\|f\|_{\mathcal{L}_{p}(\Omega)}^{p}=\int_{\Omega}|f(x)|^{p} d x \quad \text { and } \quad\|f\|_{1, \Omega}^{2}=\int_{\Omega}|\nabla f(x)|^{2}+|f(x)|^{2} d x
$$

as well as the standard $\|f\|_{\mathcal{L}_{\infty}(\Omega)}=\operatorname{ess}_{\sup _{x \in \Omega}}|f(x)|$. Due to the frequency in which the $\mathcal{L}_{2}(\Omega)$ and $\mathcal{H}^{1}(\Omega)$ norms occur, they are denoted in shorthand by $\|f\|_{0}=\|f\|_{\mathcal{L}_{2}(\Omega)}$ and $\|f\|_{1}=\|f\|_{1, \Omega}$.

The proposed change of variables is motivated by the energy law (7), which specifies the regularity of the solution: take

$$
\phi \in \mathcal{H}_{\Gamma_{D}}^{1} \equiv\left\{v \in \mathcal{H}^{1}(\Omega)|v|_{\Gamma_{D}}=\delta V\right\},
$$

and for the charge carrier concentrations,

$$
\rho_{i} \in \widetilde{W} \equiv\left\{\rho: \Omega \mapsto \mathbb{R} \mid \int_{\Omega} \rho(\log \rho-1) d x<\infty \text { and } \int_{\Omega}|\nabla \log \rho|^{2} d x<\infty\right\},
$$

leading to an implicit positivity condition. A log-transformation of the charge carrier concentrations yields a more familiar space,

$$
\eta=\log \rho \in W \equiv \mathcal{H}^{1}(\Omega) \cap \mathcal{L}^{\infty}(\Omega),
$$


and, furthermore, guarantees positivity, since $\rho=e^{\eta}>0$. As a matter of fact, in [5], it is shown for the PNP equation of two charge carriers that sufficiently regular initial data, $\eta_{i}(0) \in \mathcal{L}^{(d+1) / 2}(\Omega)$, implies that the solution $\eta_{i}(t), \phi(t)$ are in $W$ for some finite time, $0<t \leq T$, that depends on the initial data, where it is also assumed that $\partial \Omega \in C^{1+\delta}$ for some finite $\delta>0$.

\subsection{The log-density formulation and its energy}

The standard $\mathcal{L}_{2}(\Omega)$ inner-product is used

$$
(u, v)=\int_{\Omega} u v d x,
$$

and inner-products on the boundary are given by

$$
\langle u, v\rangle_{R}=\int_{\Gamma_{R}} u v d s
$$

and $\langle u, v\rangle_{N}$ is similarly defined on $\Gamma_{N} \subseteq \partial \Omega$.

Using the log-density variables, the PNP equations are written in their weak form: find $\eta_{i}(t) \in W$ with $\eta_{i, t}(t) \in \mathcal{L}_{2}(\Omega)$ and $\phi \in \mathcal{H}_{\Gamma_{D}}^{1}$ such that

$$
\begin{aligned}
(\epsilon \nabla \phi, \nabla \psi)+\langle\kappa \phi, \psi\rangle_{\Gamma_{R}}-\sum_{i=1}^{N} q_{i}\left(e^{\eta_{i}}, \psi\right) & =\langle C, \psi\rangle_{\Gamma_{R}}+\langle S, \psi\rangle_{\Gamma_{N}} \\
\left(\frac{\partial}{\partial t} e^{\eta_{i}}, w\right)+\left(D_{i} e^{\eta_{i}} \nabla\left(\eta_{i}+q_{i} \phi\right), \nabla w\right) & =0
\end{aligned}
$$

for $i=1, \ldots, N$, all $\psi \in W_{0}=\left\{\psi \in W|\psi|_{\Gamma_{D}}=0\right\}, w \in W$, and all times $0<t \leq T$, where

$$
\begin{aligned}
\left(e^{\eta_{i}(\cdot, 0)}, w\right) & =\left(e^{\eta_{i, 0}}, w\right), & \text { for all } w \in W \\
(\epsilon \nabla \phi(\cdot, 0), \nabla \psi)+\langle\kappa \phi(\cdot, 0), \psi\rangle_{\Gamma_{R}} & =\sum_{i=1}^{N} q_{i}\left(e^{\eta_{i, 0}}, \psi\right)+\langle C, \psi\rangle_{\Gamma_{R}}+\langle S, \psi\rangle_{\Gamma_{N}}, & \text { for all } \psi \in W_{0} .
\end{aligned}
$$

The energy law written in these new variables takes the form

$$
\begin{aligned}
\frac{d}{d t}\left\{\int_{\Omega} \sum_{i=1}^{N} e^{\eta_{i}}\left(\eta_{i}-1\right)+\frac{\epsilon}{2}|\nabla \phi|^{2}\right. & \left.+\sum_{i=1}^{N} q_{i} e^{\eta_{i}} \phi_{D} d x+\int_{\Gamma_{R}} \frac{\kappa}{2}|\phi|^{2} d s\right\} \\
& =-\int_{\Omega} \sum_{i=1}^{N} D_{i} e^{\eta_{i}}\left|\nabla\left(\eta_{i}+q_{i} \phi\right)\right|^{2} d x .
\end{aligned}
$$

\subsection{The discrete formulation}

Let $\mathcal{T}_{h}$ be a triangulation $(d=2)$ or tetrahedralization $(d=3)$ of the domain. For the usual space of continuous piecewise linear polynomials,

$$
W_{h} \equiv\left\{w_{h} \in \mathcal{H}^{1}(\Omega)\left|w_{h}\right|_{\tau} \in \mathbb{P}^{1} \text { for all } \tau \in \mathcal{T}_{h}\right\} \subset \mathcal{H}^{1}(\Omega),
$$


and denote the nodal interpolation operator, $\mathcal{I}_{h}: \mathcal{H}^{1}(\Omega) \rightarrow W_{h}$. When Dirichlet boundary conditions are imposed on the electrostatic potential, define the spaces of continuous piecewise linear finite element functions

$$
\begin{aligned}
W_{h, \Gamma_{D}} & \equiv\left\{\psi_{h} \in W_{h}\left|\psi_{h}\right|_{\Gamma_{D}}=\mathcal{I}_{h}(\delta V)\right\}, \\
W_{h, 0} & \equiv\left\{\psi_{h} \in W_{h}\left|\psi_{h}\right|_{\Gamma_{D}}=0\right\} .
\end{aligned}
$$

When Robin boundary conditions are imposed, the lumped boundary innerproduct,

$$
\langle u, v\rangle_{R, h}=\int_{\Gamma_{R}} \mathcal{I}_{h}(u v) d s,
$$

is needed to preserve monotonicity of the discrete Poisson equation. For the time discretization, define a partition of the time domain,

$$
0=t_{0}<t_{1}<\cdots<t_{m}=T
$$

where $\Delta t_{j} \equiv t_{j}-t_{j-1}$.

The finite element solution to the PNP equations is defined using the above finite element spaces and an implicit time discretization defined on the time partition: find $\eta_{i, h}^{(j)} \in W_{h}$ and $\phi_{h}^{(j)} \in W_{h, \Gamma_{D}}$ satisfying

$$
\begin{gathered}
\left(\epsilon \nabla \phi_{h}^{(j)}, \nabla \psi_{h}\right)+\left\langle\kappa \phi_{h}^{(j)}, \psi_{h}\right\rangle_{R, h}-\sum_{i=1}^{N} q_{i}\left(e^{\eta_{i, h}^{(j)}}, \psi_{h}\right)=\left\langle C, \psi_{h}\right\rangle_{R, h}+\left\langle S, \psi_{h}\right\rangle_{\Gamma_{N}} \\
\frac{1}{\Delta t_{j}}\left(e^{\eta_{i, h}^{(j)}}, w_{h}\right)+\left(D_{i} e^{\eta_{i, h}^{(j)}} \nabla\left(\eta_{i, h}^{(j)}+q_{i} \phi_{h}^{(j)}\right), \nabla w_{h}\right)=\frac{1}{\Delta t_{j}}\left(e^{\eta_{i, h}^{(j-1)}}, w_{h}\right),
\end{gathered}
$$

for $i=1, \ldots, N$ and all $\psi_{h} \in W_{h, 0}, w_{h} \in W_{h}$, and $j=1, \ldots, m$. The initial condition is given by

$$
\left(e^{\eta_{i, h}^{(0)}}, w_{h}\right)=\left(e^{\eta_{i, 0}}, w_{h}\right), \quad \text { for all } w_{h} \in W_{h},
$$

for $i=1, \ldots, N$ and

$$
\begin{aligned}
\left(\epsilon \nabla \phi_{h}^{(0)}, \nabla \psi_{h}\right)+\left\langle\kappa \phi_{h}^{(0)}, \psi_{h}\right\rangle_{R, h}= & \sum_{i=1}^{N} q_{i}\left(e^{\eta_{i, 0}}, \psi_{h}\right) \\
& +\left\langle C, \psi_{h}\right\rangle_{R, h}+\left\langle S, \psi_{h}\right\rangle_{\Gamma_{N}} \text { for all } \psi_{h} \in W_{h, 0} .
\end{aligned}
$$

\subsection{A discrete maximum principle}

The presence of a inhomogeneous Dirichlet boundary condition imposes additional constraints on the finite element mesh in order to maintain a discrete 
maximum principle for $\phi_{h}$. Since the boundary condition is assumed to be independent of time, the electrostatic potential can be decomposed into two terms, $\phi_{h}=\phi_{h, 0}+\phi_{h, D}$, with $\phi_{h, 0} \in W_{h, 0}$ and $\phi_{h, D} \in W_{h, \Gamma_{D}}$, where $\phi_{h, D}$ satisfies (9) with the charge densities and the terms on the right set to zero. To ensure that the energy is bounded from below, the function $\phi_{h, D}$ must satisfy some $\mathcal{L}_{\infty}(\Omega)$ estimate.

To verify the $\mathcal{L}_{\infty}$-stability, it is sufficient to show that the finite element mesh yields a monotone operator for the Poisson equation (9). Consider an element, $\tau \in \mathcal{T}_{h}$. The term facet is used below to denote an element edge when $d=2$, and an element face when $d=3$. Let $E$ be an edge (one-dimensional sub-simplex) of $\tau$. The $d-2$ dimensional simplex in $\tau$ that is opposite to the edge, $E$, is denoted by $k_{E}^{\tau}$. (In two-dimensions, $\left|k_{E}^{\tau}\right|=1$.) The angle, $\theta_{E}^{\tau}$, is the angle between the facets containing edge $E$. The average value of $\epsilon$ on element $\tau$ is denoted by $\langle\epsilon\rangle_{\tau}=\int_{\tau} \epsilon d x /|\tau|$. In [44], it was shown that the off-diagonal entries of the stiffness matrix corresponding to the vertices on edge $E$ are given by,

$$
\omega_{E} \equiv \frac{1}{d(d-1)} \sum_{\tau \supset E}\langle\epsilon\rangle_{\tau}\left|k_{E}^{\tau}\right| \cot \theta_{E}^{\tau} \geq 0,
$$

where the summation $\sum_{\tau \supset E}$ is taken over all elements $\tau \in \mathcal{T}_{h}$ containing edge $E$. In the case where $\epsilon$ is constant, this condition simply requires $\mathcal{T}_{h}$ to be a Delaunay mesh. Using this identity, a necessary and sufficient condition is given for the Poisson matrix to be monotone, implying that it has a nonnegative inverse and, consequently, a discrete maximum principle holds.

Lemma 1. On each edge, $E \in \mathcal{T}_{h}$, suppose the inequality (13) holds. Then, the finite element approximation, $\phi_{h, D} \in W_{h, \Gamma_{D}}$ defined by

$$
\left(\epsilon \nabla \phi_{h, D}, \nabla \psi_{h}\right)+\left\langle\phi_{h, D}, \psi_{h}\right\rangle_{R, h}=0 \quad \text { for all } \psi_{h} \in W_{h, 0},
$$

satisfies a discrete maximum principle

$$
\left\|\phi_{h, D}\right\|_{\mathcal{L}_{\infty}(\Omega)} \leq \sup _{x \in \Gamma_{D}}|\delta V(x)|
$$

This result comes from $[22,44]$ directly. It is important to mention that any bound

$$
\left\|\phi_{h, D}\right\|_{\mathcal{L}_{\infty}(\Omega)} \leq C_{\infty} \sup _{x \in \Gamma_{D}}|\delta V(x)|
$$

is sufficient for the energy estimate, such as the bound from [39], which only requires quasi-uniformity of the finite element mesh under some additional constraints on the coefficients of the pde. However, the energy estimate below makes explicit use of Lemma 1 to shorten the presentation of the result.

\subsection{A discrete energy estimate}

For autonomous boundary conditions, $\delta V, S$, and $C$, the stability properties of the finite element solution analogous to (6) and (7) are verified. Furthermore, if a maximum principle holds for the finite element solution to the Poisson equation, the following result holds. 
Theorem 2. Suppose $\eta_{i, h}^{(j)} \in W_{h}$ and $\phi_{h}^{(j)} \in W_{h, \Gamma_{D}}$ satisfy equations (9)-(12) for $j=1, \ldots, m$ and that (13) is satisfied. Then, the mass is conserved for each charge carrier,

$$
\int_{\Omega} e^{\eta_{i, h}^{(j)}(x, t)} d x=\int_{\Omega} e^{\eta_{i, 0}(x)} d x, \quad \text { for } i=1, \ldots, N, \quad j=1, \ldots, m,
$$

and the energy estimate is satisfied,

$$
\begin{gathered}
\int_{\Omega} \sum_{i=1}^{N} e^{\eta_{i, h}^{(m)}}\left(\eta_{i, h}^{(m)}-1\right)+\frac{\epsilon}{2}\left|\nabla \phi_{h}^{(m)}\right|^{2} d x+\frac{1}{2} \int_{\Gamma_{R}} \mathcal{I}_{h}\left(\kappa\left(\phi_{h}^{(m)}\right)^{2}\right) d s \\
+\sum_{j=1}^{m} \Delta t_{j} \int_{\Omega} \sum_{i=1}^{N} D_{i} e^{\eta_{i, h}^{(j)}}\left|\nabla\left(\eta_{i, h}^{(j)}+q_{i} \phi_{h}^{(j)}\right)\right|^{2} d x \\
\leq \int_{\Omega} \sum_{i=1}^{N} e^{\eta_{i, h}^{(0)}}\left(\eta_{i, h}^{(0)}-1\right)+\frac{\epsilon}{2}\left|\nabla \phi_{h}^{(0)}\right|^{2} d x+\frac{1}{2} \int_{\Gamma_{R}} \mathcal{I}_{h}\left(\kappa\left(\phi_{h}^{(0)}\right)^{2}\right) d s+C_{1},
\end{gathered}
$$

where $C_{1}$ depends on the number of charge carrying species, their initial masses, the electric permittivity and capacitance coefficients, but not on $T$. In the cases of no Dirichlet boundary conditions or homogeneous Dirichlet boundary conditions on $\phi_{h}$, the constant $C_{1}$ vanishes.

Proof. To prove the conservation of mass for the charged species, choose $w_{h} \equiv 1 \in W_{h}$ in equation (10) to show

$\frac{1}{\Delta t_{j}} \int_{\Omega} e^{\eta_{i, h}^{(j)}}-e^{\eta_{i, h}^{(j-1)}} d x=\left(\frac{e^{\eta_{i, h}^{(j)}}-e^{\eta_{i, h}^{(j-1)}}}{\Delta t_{j}}, 1\right)+\left(D_{i} e^{\eta_{i, h}^{(j)}} \nabla\left(\eta_{i, h}^{(j)}+q_{i} \phi_{h}^{(j)}\right), \nabla 1\right)=0$,

which yields (14). This argument expectedly fails when Dirichlet boundary conditions are imposed on $\eta_{i, h}$, since $1 \notin W_{h}$. This is not an artifact of the discretization, however, and is similar for the continuous system.

To prove the energy estimate, set $w_{h}=\eta_{i, h}^{(j)}+q_{i} \phi_{h}^{(j)} \in W_{h}$, which is a valid choice for the test function since $\phi_{h}^{(j)} \in W_{h, \Gamma_{D}} \subseteq W_{h}$. This gives

$$
\left(\frac{e^{\eta_{i, h}^{(j)}}-e^{\eta_{i, h}^{(j-1)}}}{\Delta t_{j}}, \eta_{i, h}^{(j)}\right)+q_{i}\left(\frac{e^{\eta_{i, h}^{(j)}}-e^{\eta_{i, h}^{(j-1)}}}{\Delta t_{j}}, \phi_{h}^{(j)}\right)=-\int_{\Omega} D_{i} e^{\eta_{i, h}^{(j)}}\left|\nabla\left(\eta_{i, h}^{(j)}+q_{i} \phi_{h}^{(j)}\right)\right|^{2} d x
$$

which is summed over $i=1, \ldots, N$, to get

$$
\begin{aligned}
\sum_{i=1}^{N}\left(\frac{e^{\eta_{i, h}^{(j)}}-e^{\eta_{i, h}^{(j-1)}}}{\Delta t_{j}}, \eta_{i, h}^{(j)}\right) & +\sum_{i=1}^{N} q_{i}\left(\frac{e^{\eta_{i, h}^{(j)}}-e^{\eta_{i, h}^{(j-1)}}}{\Delta t_{j}}, \phi_{h}^{(j)}\right) \\
& =-\sum_{i=1}^{N} \int_{\Omega} D_{i} e^{\eta_{i, h}^{(j)}}\left|\nabla\left(\eta_{i, h}^{(j)}+q_{i} \phi_{h}^{(j)}\right)\right|^{2} d x .
\end{aligned}
$$


The first terms on the left are bounded by using the convexity of the function $f(\rho)=\rho(\log \rho-1)$ for $\rho>0$, which can be used to show

$$
\left(\rho_{j}-\rho_{j-1}\right) \log \rho_{j} \geq \rho_{j}\left(\log \rho_{j}-1\right)-\rho_{j-1}\left(\log \rho_{j-1}-1\right) .
$$

This follows from $f^{\prime}(\rho)=\log \rho, f^{\prime \prime}(\rho)=1 / \rho>0$, and Taylor expansion. Applying this bound with $\rho_{j}=e^{\eta_{i, h}^{(j)}}$ and $\rho_{j-1}=e^{\eta_{i, h}^{(j-1)}}$, one obtains for each $i$

$$
\left(\frac{e^{\eta_{i, h}^{(j)}}-e^{\eta_{i, h}^{(j-1)}}}{\Delta t_{j}}, \eta_{i, h}^{(j)}\right) \geq \frac{\left(e^{\eta_{i, h}^{(j)}}, \eta_{i, h}^{(j)}-1\right)-\left(e^{\eta_{i, h}^{(j-1)}}, \eta_{i, h}^{(j-1)}-1\right)}{\Delta t_{j}}
$$

To bound the remaining term on the left side of (16), decompose $\phi_{h}^{(j)}=\phi_{h, 0}^{(j)}+\phi_{h, D}$, where $\phi_{h, 0}^{(j)} \in W_{h, 0}$ and $\phi_{h, D} \in W_{h, \Gamma_{D}}$ satisfies the steady differential equation subject to the interpolated Dirichlet boundary condition:

$$
\left(\epsilon \nabla \phi_{h, D}, \nabla \psi_{h}\right)+\left\langle\kappa \phi_{h, D}, \psi_{h}\right\rangle_{R, h}=0,\left.\quad \phi_{h, D}\right|_{\Gamma_{D}}=\mathcal{I}_{h}(\delta V),
$$

for all $\psi_{h} \in W_{h, 0}$. Write

$$
\sum_{i=1}^{N} q_{i}\left(\frac{e^{\eta_{i, h}^{(j)}}-e^{\eta_{i, h}^{(j-1)}}}{\Delta t_{j}}, \phi_{h}^{(j)}\right)=\sum_{i=1}^{N} q_{i}\left(\frac{e^{\eta_{i, h}^{(j)}}-e^{\eta_{i, h}^{(j-1)}}}{\Delta t_{j}}, \phi_{h, 0}^{(j)}\right)+\sum_{i=1}^{N} q_{i}\left(\frac{e^{\eta_{i, h}^{(j)}}-e^{\eta_{i, h}^{(j-1)}}}{\Delta t_{j}}, \phi_{h, D}\right)
$$

and bound the first term on the right by subtracting consecutive time-steps of (9) and taking $\psi_{h}=\phi_{h, 0}^{(j)} \in W_{h, 0}$,

$$
\begin{aligned}
& \sum_{i=1}^{N} q_{i}\left(\frac{e^{\eta_{i, h}^{(j)}}-e^{\eta_{i, h}^{(j-1)}}}{\Delta t_{j}}, \phi_{h, 0}^{(j)}\right)=\left(\epsilon \frac{\nabla \phi_{h, 0}^{(j)}-\nabla \phi_{h, 0}^{(j-1)}}{\Delta t_{j}}, \nabla \phi_{h, 0}^{(j)}\right)+\left\langle\kappa \frac{\phi_{h, 0}^{(j)}-\phi_{h, 0}^{(j-1)}}{\Delta t_{j}}, \phi_{h, 0}^{(j)}\right\rangle_{R, h} \\
&=\left(\epsilon \frac{\nabla \phi_{h}^{(j)}-\nabla \phi_{h}^{(j-1)}}{\Delta t_{j}}, \nabla \phi_{h}^{(j)}\right)+\left\langle\kappa \frac{\phi_{h}^{(j)}-\phi_{h}^{(j-1)}}{\Delta t_{j}}, \phi_{h}^{(j)}\right\rangle_{R, h} \\
& \geq \frac{\left(\epsilon \nabla \phi_{h}^{(j)}, \nabla \phi_{h}^{(j)}\right)-\left(\epsilon \nabla \phi_{h}^{(j-1)}, \nabla \phi_{h}^{(j-1)}\right)}{2 \Delta t_{j}} \quad(19) \\
&+\frac{\left\langle\kappa \phi_{h}^{(j)}, \phi_{h}^{(j)}\right\rangle_{R, h}-\left\langle\kappa \phi_{h}^{(j-1)}, \phi_{h}^{(j-1)}\right\rangle_{R, h}}{2 \Delta t_{j}},
\end{aligned}
$$

where the second equality follows from adding and subtracting the term

$$
\Delta t_{i}^{-1}\left[\left(\epsilon \nabla \phi_{h, D}, \nabla \phi_{h, 0}^{(j)}\right)+\left\langle\kappa \phi_{h, D}, \phi_{h, 0}^{(j)}\right\rangle_{R, h}\right]=0 .
$$

Combining (16) - (19) gives the bound

$$
\begin{aligned}
& \frac{\left[\mathcal{E}_{h}^{(j)}+\sum_{i=1}^{N} q_{i}\left(e^{\eta_{i, h}^{(j)}}, \phi_{h, D}\right)\right]}{-\left[\mathcal{E}_{h}^{(j-1)}+\sum_{i=1}^{N} q_{i}\left(e^{\eta_{i, h}^{(j-1)}}, \phi_{h, D}\right)\right]} \Delta t_{j} \\
& \leq-\sum_{i=1}^{N} \int_{\Omega} D_{i} e^{\eta_{i, h}^{(j)}}\left|\nabla\left(\eta_{i, h}^{(j)}+q_{i} \phi_{h}^{(j)}\right)\right|^{2} d x,
\end{aligned}
$$


where $\mathcal{E}_{h}^{(k)}$ denotes the discrete energy functional,

$$
\mathcal{E}_{h}^{(k)} \equiv \int_{\Omega} \sum_{i=1}^{N} e^{\eta_{i, h}^{(k)}}\left(\eta_{i, h}^{(k)}-1\right)+\frac{\epsilon}{2}\left|\nabla \phi_{h}^{(k)}\right|^{2} d x+\frac{1}{2} \int_{\Gamma_{R}} \mathcal{I}_{h}\left(\kappa\left(\phi_{h}^{(k)}\right)^{2}\right) d s .
$$

The left side of (20) yields a telescoping sum; a summation over $j$ leads to

$$
\begin{array}{r}
{\left[\mathcal{E}_{h}^{(m)}+\sum_{i=1}^{N} q_{i}\left(e^{\eta_{i, h}^{(m)}}, \phi_{h, D}\right)\right]+\sum_{j=1}^{m} \Delta t_{j} \sum_{i=1}^{N} \int_{\Omega} D_{i} e^{\eta_{i, h}^{(j)}}\left|\nabla\left(\eta_{i, h}^{(j)}+q_{i} \phi_{h}^{(j)}\right)\right|^{2} d x} \\
\leq \mathcal{E}_{h}^{(0)}+\sum_{i=1}^{N} q_{i}\left(e^{\eta_{i, h}^{(0)}}, \phi_{h, D}\right) .
\end{array}
$$

To complete the proof, the conservation of mass bounds

$$
\begin{aligned}
\sum_{i=1}^{N} q_{i}\left(e^{\eta_{i, h}^{(0)}}-e^{\eta_{i, h}^{(m)}}, \phi_{h, D}\right) \leq & 2 \sum_{i=1}^{N}\left\|q_{i} e^{\eta_{i, h}^{(0)}}\right\|_{\mathcal{L}^{1}(\Omega)}\left\|\phi_{h, D}\right\|_{\mathcal{L}_{\infty}(\Omega)} \\
& \leq 2 N\left(\max _{1 \leq i \leq N}\left\|q_{i} e^{\eta_{i, h}^{(0)}}\right\|_{\mathcal{L}^{1}(\Omega)}\right)\left\|\phi_{h, D}\right\|_{\mathcal{L}_{\infty}(\Omega)},
\end{aligned}
$$

where $\left\|q_{i} e^{\eta_{i, h}^{(0)}}\right\|_{\mathcal{L}^{1}(\Omega)}$ is directly proportional to the charge carrier's mass, determined by the initial condition. The estimate,

$$
\left\|\phi_{h, D}\right\|_{\mathcal{L}_{\infty}(\Omega)} \leq \max _{x \in \Gamma_{D}}\left|\mathcal{I}_{h}(\delta V)\right|
$$

follows from Lemma 1. In the case where $\delta V \equiv 0$ or $\Gamma_{D}=\emptyset$, it is clear that $\phi_{h, D} \equiv 0$ so that this term vanishes altogether. As a final note, since the Dirichlet boundary term fits in with the telescoping terms in (20), it is not proportional to the length of the time domain.

To conclude this section, one important remark is in order. The inequality in this energy estimate is a consequence of only two aspects of the discretization; first, the time discretization satisfies (17) and (19) only with an inequality, whereas the semi-discrete solution (continuous in time) satisfies these bounds with equality. The only other inequality in the proof of Theorem 2 is used to bound inhomogeneous Dirichlet boundary conditions. As a matter of fact, in the semi-discrete case with homogeneous Dirichlet boundary conditions (or no Dirichlet boundary conditions), the finite element solution satisfies the energy estimate with equality.

\section{Electrokinetics}

Electrokinetic systems combine effects of electrostatic systems coupled with incompressible fluid flow. The model equations studied here couple the PNP equations with the incompressible Navier-Stokes (NS) equations. This system of 
equations models electrokinetic phenomena such as electroosmosis, electrophoresis, streaming potentials, electrowetting, and many other phenomena where charged particles and a charged fluid interact $[11,21,24,40]$. Some analysis for this system in the continuous case is carried out in [36]. The equations governing the electrokinetic system seek a solution comprised of the charge carrier log-densities, $\eta_{1}, \ldots, \eta_{N}$, the electrostatic potential, $\phi$, the fluidic velocity, $\vec{u}$, and the fluidic pressure, $p$, that satisfy the equations

$$
\begin{aligned}
-\nabla \cdot(\epsilon \nabla \phi) & =\sum_{i=1}^{N} q_{i} e^{\eta_{i}} \\
\frac{\partial}{\partial t} e^{\eta_{i}} & =\nabla \cdot\left(D_{i} e^{\eta_{i}} \nabla\left(\eta_{i}+q_{i} \phi\right)-e^{\eta_{i}} \vec{u}\right), \quad i=1, \ldots, N, \\
\rho_{f}\left(\vec{u}_{t}+(\vec{u} \cdot \nabla) \vec{u}\right)+\nabla p & =\nabla \cdot(2 \mu \varepsilon(\vec{u}))-\sum_{i=1}^{N} q_{i} e^{\eta_{i}} \nabla \phi, \\
\nabla \cdot \vec{u} & =0
\end{aligned}
$$

on $\Omega \times(0, T]$, where $\varepsilon(\cdot)$ denotes the symmetrized vector gradient,

$$
\varepsilon(\vec{u})=\frac{1}{2}\left(\nabla \vec{u}+(\nabla \vec{u})^{T}\right) .
$$

The initial conditions for this system are given for $x \in \Omega$,

$$
\eta_{i}(x, 0)=\eta_{i, 0}(x), \quad-\nabla \cdot(\epsilon \nabla \phi(x, 0))=\sum_{i=1}^{N} q_{i} e^{\eta_{i, 0}(x)}, \quad \vec{u}(x, 0)=\vec{u}_{0}(x),
$$

where $\nabla \cdot \vec{u}_{0}=0$ in $\Omega$ and $p(\cdot, 0)$ is the pressure corresponding to the initial fluid velocity field. Equations (22) and (23) come directly from the PNP model, where an additional coupling term in (23) models a kinetic force from the fluid flow described by the NS equations.

Equations (24) and (25) are the usual NS equations for an incompressible fluid. In (24), the coefficient $\rho_{f}$ denotes the fluid density, assumed to be constant, and $\mu$ denotes the fluid viscosity. The term, $\sum_{i=1}^{N} q_{i} e^{\eta_{i}} \nabla \phi$, in (24) models the electrostatic body force acting on the fluid.

\subsection{Boundary conditions}

The boundary conditions considered for the PNP variables remain the same as the previous section (4)-(5). The NS boundary conditions are assumed to be some combination of no-flux and no-slip boundary conditions. Again, this corresponds to an energetically closed system. Take $\Gamma_{\text {no-flux }} \subseteq \partial \Omega$ and $\Gamma_{\text {no-slip }}=\partial \Omega \backslash \Gamma_{\text {no-flux }}$. Then, for $\vec{n}$ the outward unit normal vector and $\vec{t}$ any unit tangent vector to $\partial \Omega$, the fluid velocity must satisfy

$$
\begin{aligned}
\vec{u} \cdot \vec{n} & =0, & & \text { on } \Gamma_{\text {no-flux }}, \\
(\mu \varepsilon(\vec{u}) \vec{n}) \cdot \vec{t} & =0, & & \text { on } \Gamma_{\text {no-flux }}, \\
\vec{u} & =\overrightarrow{0}, & & \text { on } \Gamma_{\text {no-slip }} .
\end{aligned}
$$


Due to the incompressibility condition on the fluid velocity (25), the solution satisfies $\varepsilon(\vec{u})=\nabla \vec{u}$, which is commonly used to represent the viscosity term in the continuity equation (24). This identity does not hold, however, for general $\vec{v} \in\left[\mathcal{H}^{1}(\Omega)\right]^{d}$, so one must take care when writing the PNP-NS system in weak form; namely, for $\vec{v} \cdot \vec{n}=0$ on $\partial \Omega$, the divergence theorem gives

$$
-(\nabla \cdot(2 \mu \varepsilon(\vec{u})), \vec{v})=(2 \mu \varepsilon(\vec{u}), \varepsilon(\vec{v})) .
$$

In the special case when $\vec{u}, \vec{v} \equiv \overrightarrow{0}$ on $\partial \Omega$, the right side reduces to $(\mu \nabla \vec{u}, \nabla \vec{v})$.

\subsection{Energy of the electrokinetic system}

The corresponding energy law for this system is given by

$$
\begin{aligned}
\frac{d}{d t}\left\{\int_{\Omega} \frac{\rho_{f}}{2}|\vec{u}|^{2}+\sum_{i=1}^{N}\right. & \left.\rho_{i}\left(\log \rho_{i}-1\right)+\frac{\epsilon}{2}|\nabla \phi|^{2}+\sum_{i=1}^{N} q_{i} \rho_{i} \phi_{D} d x+\int_{\Gamma_{R}} \frac{\kappa}{2}|\phi|^{2} d s\right\} \\
& =-\int_{\Omega} \frac{\mu}{2}|\varepsilon(\vec{u})|^{2}+\sum_{i=1}^{N} D_{i} \rho_{i}\left|\nabla\left(\log \rho_{i}+q_{i} \phi\right)\right|^{2} d x
\end{aligned}
$$

The terms in the energy law relating to the NS variables are critically hinged on specific mathematical structures of the NS system. In particular, the divergencefree property of the fluidic velocity plays a significant role in the cancelation of the cross-terms between the PNP and NS systems. As a result, the discrete solution should satisfy the divergence-free property on every subdomain of $\Omega$. This can be accomplished in several ways, using higher order elements or discontinuous Galerkin (DG) approximations [1, 8], for example. In many practical applications, solutions using higher order elements may be prohibitively expensive to compute; the discussion below primarily considers DG approximations for the NS variables.

\subsection{Weak formulation for the NS system}

To define the weak solution to the NS equations, define

$$
\begin{aligned}
& Q \equiv \mathcal{L}_{2}(\Omega) \\
& V \equiv\left\{\vec{v} \in\left[\mathcal{H}^{1}(\Omega)\right]^{d} \mid \vec{v} \cdot \vec{n}=0 \text { on } \Gamma_{\text {no-flux }}, \quad \vec{u}=\overrightarrow{0} \text { on } \Gamma_{\text {no-slip }}\right\}, \\
& \mathcal{H}_{D, 0}(\operatorname{div} ; \Omega) \equiv\left\{\vec{v} \in\left[\mathcal{L}_{2}(\Omega)\right]^{d} \mid \nabla \cdot \vec{v} \in \mathcal{L}_{2}(\Omega), \quad \vec{v} \cdot \vec{n}=0 \text { on } \Gamma_{\text {no-flux }},\right. \\
&\left.\vec{u}=\overrightarrow{0} \text { on } \Gamma_{\text {no-slip }}\right\} .
\end{aligned}
$$

The discussion of the weak formulation of the NS equations requires no special treatment for the PNP variables; it is convenient to replace the electrostatic body force by some generic function, $\vec{f} \in\left[\mathcal{L}_{2}(\Omega)\right]^{d}$. The weak solution of the NS equations is $(\vec{u}, p) \in V \times Q$ satisfying

$$
\begin{aligned}
D_{t}(\vec{u} ; \vec{u}, \vec{v})+A(\vec{u}, \vec{v})+B(\vec{v}, p) & =(\vec{f}, \vec{v}), & & \text { for all } \vec{v} \in V, \\
B(\vec{u}, q) & =0, & & \text { for all } q \in Q,
\end{aligned}
$$


where

$$
\begin{aligned}
D_{t}(\vec{w} ; \vec{u}, \vec{v}) & \equiv \rho_{f}\left(\vec{u}_{t}, \vec{v}\right)+\rho_{f}((\vec{w} \cdot \nabla) \vec{u}, \vec{v}), \\
A(\vec{u}, \vec{v}) & \equiv(2 \mu \varepsilon(\vec{u}), \varepsilon(\vec{v})), \\
B(\vec{u}, q) & \equiv-(\nabla \cdot \vec{u}, q) .
\end{aligned}
$$

The well-posedness of the weak formulation can be demonstrated following [12], where a Korn inequality must be established. The following estimate is a statement of the Korn inequality and is exactly Lemma 2.1 in [1].

Lemma 3. Let $\Omega \subset \mathbb{R}^{d}, d=2,3$ be a polygonal or polyhedral domain. Then, there exists a positive constant $C_{K}$ (depending on the domain through its diameter and shape) such that

$$
|\vec{v}|_{1} \leq C_{K}\|\varepsilon(\vec{v})\|_{0}, \quad \text { for all } \vec{v} \in V .
$$

\subsection{Some div-conforming, discontinuous finite element pairs}

Recall that $\mathcal{T}_{h}$ denotes the finite element mesh on $\Omega$ and let $\mathcal{F}_{h}$ denote the set of interior element facets. The broken $\mathcal{L}_{2}$ and $\mathcal{H}^{1}$ inner-products and norms are defined in the usual way

$$
(p, q)_{\mathcal{T}_{h}} \equiv \sum_{\tau \in \mathcal{T}_{h}}(p, q)_{\tau}, \quad\|q\|_{0, \mathcal{T}_{h}} \equiv(q, q)_{\mathcal{T}_{h}}^{1 / 2}, \quad \text { and } \quad|s|_{1, \mathcal{T}_{h}} \equiv(\nabla s, \nabla s)_{\mathcal{T}_{h}}^{1 / 2},
$$

for $p, q \in \mathcal{L}_{2}(\Omega)$ and $s \in \mathcal{H}^{1}\left(\mathcal{T}_{h}\right) \equiv\left\{s \in \mathcal{L}_{2}(\Omega)|s|_{\tau} \in \mathcal{H}^{1}(\tau)\right.$ for all $\left.\tau \in \mathcal{T}_{h}\right\}$.

Let $w \in \mathcal{H}^{1}\left(\mathcal{T}_{h}\right), \vec{v} \in\left[\mathcal{H}^{1}\left(\mathcal{T}_{h}\right)\right]^{d}, \sigma \in\left[\mathcal{H}^{1}\left(\mathcal{T}_{h}\right)\right]^{d \times d}$ denote a scalar, vector, and rank-two tensor field, respectively. These fields are $\mathcal{H}^{1}$-regular within each element, though inter-element continuity is not assumed. Fix $e \in \mathcal{F}_{h}$, where $e=\tau_{+} \cap \tau_{-}$. Denote the outward unit normal vectors of $\tau_{+}$and $\tau_{-}$by $\vec{n}_{+}$and $\vec{n}_{-}$, respectively; the averages across $e$ on internal facets are defined by

$$
\{w\} \equiv \frac{1}{2}\left(w_{+}+w_{-}\right), \quad\{\vec{v}\} \equiv \frac{1}{2}\left(\vec{v}_{+}+\vec{v}_{-}\right), \quad \text { and } \quad\{\sigma\} \equiv \frac{1}{2}\left(\sigma_{+}+\sigma_{-}\right),
$$

and given by their traces on the boundary facets; the jumps across internal facets are given by

$$
\begin{aligned}
\llbracket w \rrbracket & \equiv w_{+} \vec{n}_{+}+w_{-} \vec{n}_{-}, & & {[\vec{v}] \equiv \vec{v}_{+} \cdot \vec{n}_{+}+\vec{v}_{-} \cdot \vec{n}_{-}, } \\
\llbracket \vec{v} \rrbracket & \equiv \vec{v}_{+} \otimes \vec{n}_{+}+\vec{v}_{-} \otimes \vec{n}_{-}, & & {[\sigma] \equiv \sigma_{+} \vec{n}_{+}+\sigma_{-} \vec{n}_{-}, }
\end{aligned}
$$

and $\llbracket w \rrbracket=w \vec{n},[\vec{v}]=\vec{v} \cdot \vec{n}, \llbracket \vec{v} \rrbracket=\vec{v} \otimes \vec{n}$, and $[\sigma]=\sigma \vec{n}$ on boundary facets. The subscripts on the functions are equipped with their natural meanings of restriction to the element $\tau_{+}$or $\tau_{-}$. An inner-product on the inter-element facets is defined,

$$
\langle w, v\rangle_{\mathcal{F}_{h}} \equiv \sum_{e \in \mathcal{F}_{h}} \int_{e} w(s) v(s) d s
$$


To motivate the bilinear form of the DG approach, assume $\vec{u}, \vec{v} \in \mathcal{H}^{2}\left(\mathcal{T}_{h}\right) \cap V$. Then,

$$
\begin{gathered}
-\int_{\Omega} \nabla \cdot(2 \mu \nabla \varepsilon(\vec{u})) \cdot \vec{v} d x=\sum_{\tau \in \mathcal{T}_{h}} \int_{\tau} 2 \mu \varepsilon(\vec{u}): \varepsilon(\vec{v}) d x-\sum_{\tau \in \mathcal{T}_{h}} \int_{\partial \tau} 2 \mu\left(\varepsilon(\vec{u}) \vec{n}_{\tau}\right) \cdot \vec{v} d s \\
=(2 \mu \varepsilon(\vec{u}), \varepsilon(\vec{v}))_{\mathcal{T}_{h}}-\langle 2 \mu \llbracket \vec{u} \rrbracket,\{\varepsilon(\vec{v})\}\rangle_{\mathcal{F}_{h}}-\langle 2 \mu\{\varepsilon(\vec{u})\}, \llbracket \vec{v} \rrbracket\rangle_{\mathcal{F}_{h}} .
\end{gathered}
$$

It is straightforward to check that the solution of (27)-(28) also solves the variational problem with $A(\cdot, \cdot)$ replaced by the above expression.

To preserve the local divergence-free property of the fluid velocity, nonconforming finite elements are useful for assigning degrees of freedom aimed at preserving this property instead of conforming to the continuous spaces. The finite element spaces are defined for the pressure, $Q_{h} \subset Q$, and for the fluid velocity, $V_{h} \subset\left[\mathcal{H}^{1}\left(\mathcal{T}_{h}\right)\right]^{d} \cap \mathcal{H}_{D, 0}(\operatorname{div})$, where $V_{h} \not \subset V$, in general. While it is not necessary that $V_{h}$ conforms to $V$, several constraints are imposed on the finite element pair $V_{h} \times Q_{h}$ to ensure the well-posedness of the discrete problem and the divergence-free property of the discrete fluid velocity. First, it is required that the finite element pair is div-conforming, meaning

$$
\nabla \cdot V_{h} \subseteq Q_{h}
$$

and, second, that there exists for each $q_{h} \in Q_{h}$ a corresponding $\vec{v}_{h} \in V_{h}$ such that

$$
\nabla \cdot \vec{v}_{h}=q_{h} \quad \text { and } \quad\left\|\vec{v}_{h}\right\|_{0} \leq c_{P}\left\|q_{h}\right\|_{0},
$$

where $c_{P}>0$ is a Poincaré constant that depends on $\Omega$ in general, but not on $q_{h}$. Requirements (30) and (31) together imply that $\nabla \cdot V_{h}=Q_{h}$. The final requirement for well-posedness is the existence of an interpolation operator, $\Pi_{h}: V \rightarrow V_{h}$, that satisfies the following estimates on each element, $\tau \in \mathcal{T}_{h}$ :

$$
\left|\Pi_{h} \vec{v}\right|_{1, \tau} \leq C_{\Pi}|\vec{v}|_{1, \tau} \quad \text { and } \quad\left\|\left(I-\Pi_{h}\right) \vec{v}\right\|_{0, \tau} \leq C_{\Pi}^{\prime} h_{\tau}^{s}|\vec{v}|_{1, \tau},
$$

with $h_{\tau}=\operatorname{diam}(\tau)$ and $1 \leq s \leq k+1$, where $k$ is the polynomial degree of the div-conforming element.

Some well-studied finite element pairs satisfying (30)-(32) are the RaviartThomas elements, Brezzi-Douglas-Marini elements, and the Brezzi-DouglasFortin-Marini elements, all of degree $k \geq 1$. Furthermore, as all of these elements are div-conforming, they have continuous normal components across inter-element facets, which, loosely speaking, "reduces" the discontinuity of the finite element space, requiring simpler penalty functions in the discontinuous formulation. This additional continuity also plays a role in the energy estimate of the PNP-NS system and is commented upon in the proof of Theorem 4. 


\subsection{A discrete formulation}

The discrete formulation of the NS equations is given by: find $\left(\vec{u}_{h}^{(j)}, p_{h}^{(j)}\right) \in$ $V_{h} \times Q_{h}$ such that

$$
\begin{gathered}
D_{h, t}\left(\vec{u}_{h}^{(j)} ; \vec{u}_{h}^{(j)}, \vec{v}_{h}\right)+A_{h}\left(\vec{u}_{h}^{(j)}, \vec{v}_{h}\right)+B_{h}\left(\vec{v}_{h}, p_{h}^{(j)}\right)=\frac{\rho_{f}}{\Delta t_{j}}\left(\vec{u}_{h}^{(j-1)}, \vec{v}_{h}\right)+\left(\vec{f}\left(t_{j}\right), \vec{v}_{h}\right), \\
B_{h}\left(\vec{u}_{h}^{(j)}, q_{h}\right)=0
\end{gathered}
$$

for all $\vec{v}_{h} \in V_{h}, q_{h} \in Q_{h}$, and $j=1, \ldots, m$, where the initial condition for the fluid velocity is computed by projection, $\vec{u}_{h}^{(0)}=\Pi_{h} \vec{u}_{0}$.

The forms used to define the discrete solution are given by

$$
\begin{aligned}
D_{h, t}\left(\vec{w}_{h} ; \vec{u}_{h}, \vec{v}_{h}\right) \equiv & \frac{\rho_{f}}{\Delta t_{j}}\left(\vec{u}_{h}, \vec{v}_{h}\right)-\rho_{f}\left(\left(\vec{w}_{h} \cdot \nabla\right) \vec{v}_{h}, \vec{u}_{h}\right)+\rho_{f} \sum_{\tau \in \mathcal{T}_{h}} \int_{\partial \tau}\left(\vec{w}_{h} \cdot \vec{n}_{\tau}\right)\left(\vec{u}_{h}^{w} \cdot \vec{v}_{h}\right) d s, \\
A_{h}\left(\vec{u}_{h}, \vec{v}_{h}\right) \equiv & \left(2 \mu \varepsilon\left(\vec{u}_{h}\right), \varepsilon\left(\vec{v}_{h}\right)\right)_{\mathcal{T}_{h}}-\left\langle 2 \mu\left\{\varepsilon\left(\vec{u}_{h}\right)\right\}, \llbracket \vec{v}_{h} \rrbracket\right\rangle_{\mathcal{F}_{h}}-\left\langle 2 \mu \llbracket \vec{u}_{h} \rrbracket,\left\{\varepsilon\left(\vec{v}_{h}\right)\right\}\right\rangle_{\mathcal{F}_{h}} \\
& \quad+\alpha \sum_{e \in \mathcal{F}_{h}} h_{e}^{-1} \int_{e} \mu \llbracket \vec{u}_{h} \rrbracket: \llbracket \vec{v}_{h} \rrbracket d s, \\
B_{h}\left(\vec{u}_{h}, q_{h}\right) \equiv & -\left(\nabla \cdot \vec{u}_{h}, q_{h}\right)_{\mathcal{T}_{h}} .
\end{aligned}
$$

These forms are quite standard in the DG literature, though some terms and important properties remain to be specified in the following.

The discrete kinematic derivative term, $D_{h, t}: V_{h} \times V_{h} \times V_{h} \rightarrow \mathbb{R}$, is defined using the upwind flux, $\vec{u}_{h}^{w}$, given by

$$
\vec{u}_{h}^{w}=\lim _{\delta \rightarrow 0^{+}} \vec{u}_{h}\left(x-\delta \vec{w}_{h}(x)\right) .
$$

This definition yields coercivity, summarized by the standard identity [8]: for $u_{h}, w_{h} \in V_{h}$ with $\nabla \cdot \vec{w}_{h}=0$ in $\Omega$,

$$
D_{h, t}\left(w_{h} ; \vec{u}_{h}, \vec{u}_{h}\right)=\frac{\rho_{f}}{2} \sum_{e \in \mathcal{F}_{h}} \int_{e}\left|\vec{w}_{h} \cdot \vec{n}\right|\left|\llbracket \vec{u}_{h} \rrbracket\right|^{2} d s,
$$

where $\vec{n}$ denotes either unit normal vector to the facet, $e$.

The bilinear forms, $A_{h}$ and $B_{h}$, are a standard description for a DG discretization of Stokes' equations and are motivated by the definition of the weak derivative followed by applying the divergence theorem element-wise. The parameter, $\alpha>0$, penalizes discontinuities of the solution across element interfaces and must be chosen to be sufficiently large to ensure the existence and uniqueness of the finite element solution.

Since the finite element space, $V_{h}$, is div-conforming (30), equation (34) implies that $\nabla \cdot \vec{u}_{h}=0$ on each element, $\tau \in \mathcal{T}_{h}$. Another useful property inherited from (30) is that all $\vec{v}_{h} \in V_{h}$ have continuous normal components 
across element facets; namely, letting $\vec{n}$ and $\vec{t}$ denote the normal and tangent unit vectors, respectively, on each facet, $e \in \mathcal{F}_{h}$, gives

$$
\vec{v}_{h}(x)=\left(\vec{v}_{h} \cdot \vec{n}\right) \vec{n}+\left(\vec{v}_{h} \cdot \vec{t}\right) \vec{t}=\vec{v}_{h}^{n}(x)+\vec{v}_{h}^{t}(x),
$$

and $\llbracket \vec{v}_{h}^{n} \rrbracket=0$. As a result, it holds that

$$
\int_{e} \llbracket \vec{v}_{h} \rrbracket: \sigma d s=\int_{e} \llbracket \vec{v}_{h}^{t} \rrbracket: \sigma d s \quad \text { for any } \sigma \in\left[\mathcal{H}^{1}(\Omega)\right]^{d \times d} .
$$

Using this result, the coercivity of the kinematic derivative term, $D_{h, t}$, reduces to

$$
D_{h, t}\left(\vec{w}_{h} ; \vec{u}_{h}, \vec{u}_{h}\right)=\frac{\rho_{f}}{2} \sum_{e \in \mathcal{F}_{h}} \int_{e}\left|\vec{w}_{h} \cdot \vec{n}\right|\left|\llbracket \vec{u}_{h}^{t} \rrbracket\right|^{2} d s
$$

and, for $A_{h}$,

$$
\begin{array}{r}
A_{h}\left(\vec{u}_{h}, \vec{v}_{h}\right) \equiv\left(2 \mu \varepsilon\left(\vec{u}_{h}\right), \varepsilon\left(\vec{v}_{h}\right)\right)_{\mathcal{T}_{h}}-\left\langle 2 \mu\left\{\varepsilon\left(\vec{u}_{h}\right)\right\}, \llbracket \vec{v}_{h}^{t} \rrbracket\right\rangle_{\mathcal{F}_{h}}-\left\langle 2 \mu \llbracket \vec{u}_{h}^{t} \rrbracket,\left\{\varepsilon\left(\vec{v}_{h}\right)\right\}\right\rangle_{\mathcal{F}_{h}} \\
+\alpha \sum_{e \in \mathcal{F}_{h}} h_{e}^{-1} \int_{e} \mu \llbracket \vec{u}_{h}^{t} \rrbracket: \llbracket \vec{v}_{h}^{t} \rrbracket d s,
\end{array}
$$

where only the tangent components along the element facets are penalized, and $B_{h}$ becomes

$$
B_{h}\left(\vec{v}_{h}, q_{h}\right)=-\left(\nabla \cdot \vec{v}_{h}, q_{h}\right) \quad \text { for all } \vec{v}_{h} \in V_{h} \text { and } q_{h} \in Q_{h} .
$$

The energy norm for the discontinuous fluid velocity is defined by

$$
\|\vec{v}\|_{D G}^{2}=|\vec{v}|_{1, \mathcal{T}_{h}}^{2}+|\vec{v}|_{*}^{2}, \quad \text { where } \quad|\vec{v}|_{*}^{2} \equiv \sum_{e \in \mathcal{F}_{h}} h_{e}^{-1}\left\|\llbracket \vec{v}^{t} \rrbracket\right\|_{0, e}^{2} .
$$

For any of the three finite element examples mentioned above, one can establish the $\|\cdot\|_{D G}$-stability of the bilinear form, $A_{h}$, meaning that there exists a positive constant, $\gamma$, such that

$$
A_{h}\left(\vec{v}_{h}, \vec{v}_{h}\right) \geq \gamma\left\|\vec{v}_{h}\right\|_{D G}^{2}, \quad \text { for all } \vec{v}_{h} \in V_{h} \text { (see [1]). }
$$

The stability constant, $\gamma$, depends on $\mu, \Omega$, the penalty parameter, $\alpha$, and the choice of div-conforming finite element (through the interpolation bounds in (32)). This stability, together with an argument using fixed point iteration [8], is used to verify the existence of a discrete solution for the NS equations using this DG scheme.

\subsection{The discrete electrokinetic system}

Employing the discretization of the PNP system given in the previous section and the discretization of the NS system above, the discrete solution to the 
electrokinetic system is defined by the finite element functions $\eta_{1, h}^{(j)}, \ldots, \eta_{N, h}^{(j)} \in$ $W_{h}, \phi_{h}^{(j)} \in W_{h, \Gamma_{D}}$, and $\left(\vec{u}_{h}^{(j)}, p_{h}^{(j)}\right) \in V_{h} \times Q_{h}$ satisfying

$$
\begin{aligned}
& \left(\epsilon \nabla \phi_{h}^{(j)}, \nabla \psi_{h}\right)+\left\langle\kappa \phi_{h}^{(j)}, \psi_{h}\right\rangle_{R, h}=\sum_{i=1}^{N} q_{i}\left(e^{\eta_{i, h}^{(j)}}, \psi_{h}\right) \\
& +\left\langle C^{(j)}, \psi_{h}\right\rangle_{R, h}+\left\langle S^{(j)}, \psi_{h}\right\rangle_{\Gamma_{N}}, \\
& \frac{1}{\Delta t_{j}}\left(e^{\eta_{i, h}^{(j)}}, w_{h}\right)+\left(D_{i} e^{\eta_{i, h}^{(j)}} \nabla\left(\eta_{i, h}^{(j)}+q_{i} \phi_{h}^{(j)}\right), \nabla w_{h}\right)=\frac{1}{\Delta t_{j}}\left(e^{\eta_{i, h}^{(j-1)}}, w_{h}\right)+\left(e^{\eta_{i, h}^{(j)}} \vec{u}_{h}^{(j)}, \nabla w_{h}\right), \\
& D_{h, t}\left(\vec{u}_{h}^{(j)} ; \vec{u}_{h}^{(j)}, \vec{v}_{h}\right)+A_{h}\left(\vec{u}_{h}^{(j)}, \vec{v}_{h}\right)+B_{h}\left(\vec{v}_{h}, p_{h}^{(j)}\right)=\frac{\rho_{f}}{\Delta t_{j}}\left(\vec{u}_{h}^{(j-1)}, \vec{v}_{h}\right)-\sum_{i=1}^{N} q_{i}\left(e^{\eta_{i, h}^{(j)}} \nabla \phi_{h}^{(j)}, \vec{v}_{h}\right) \\
& B_{h}\left(\vec{u}_{h}^{(j)}, q_{h}\right)=0,
\end{aligned}
$$

for all $w_{h} \in W_{h}, \psi_{h} \in W_{h, 0}, \vec{v}_{h} \in V_{h}, q_{h} \in Q_{h}$, and $j=1, \ldots, m$. Initial conditions are prescribed by

$$
\begin{aligned}
& \left(e^{\eta_{i, h}^{(0)}}, w_{h}\right)=\left(e^{\eta_{i, 0}}, w_{h}\right), \quad \text { for all } w_{h} \in W_{h}, \\
& \left(\epsilon \nabla \phi_{h}^{(0)}, \nabla \psi_{h}\right)+\left\langle\kappa \phi_{h}^{(0)}, \psi_{h}\right\rangle_{R, h}=\sum_{i=1}^{N} q_{i}\left(e^{\eta_{i, 0}}, \psi_{h}\right), \\
& +\left\langle C, \psi_{h}\right\rangle_{R, h}+\left\langle S, \psi_{h}\right\rangle_{\Gamma_{N}} \text { for all } \psi_{h} \in W_{h, 0}, \\
& \vec{u}_{h}^{(0)}=\Pi_{h} \vec{u}_{0},
\end{aligned}
$$

The stability of the discrete solution of the electrokinetic system is established in the following theorem.

Theorem 4. Suppose $\eta_{i, h}^{(j)} \in W_{h}, \phi_{h}^{(j)} \in W_{h, \Gamma_{D}}, \vec{u}_{h}^{(j)} \in V_{h}, p_{h}^{(j)}$ satisfy equations (37) - (43), where $V_{h} \times Q_{h}$ satisfies (30)-(32). Furthermore, suppose the mesh satisfies the hypothesis of Lemma 1. Then, the mass is conserved for each charge carrier,

$$
\int_{\Omega} e^{\eta_{i, h}^{(j)}(x, t)} d x=\int_{\Omega} e^{\eta_{i, 0}(x)} d x, \quad \text { for } i=1, \ldots, N, \quad j=1, \ldots, m,
$$


and the energy estimate is satisfied,

$$
\begin{gathered}
\int_{\Omega} \frac{\rho_{f}}{2}\left|\vec{u}_{h}^{(m)}\right|^{2}+\sum_{i=1}^{N} e^{\eta_{i, h}^{(m)}}\left(\eta_{i, h}^{(m)}-1\right)+\frac{\epsilon}{2}\left|\nabla \phi_{h}^{(m)}\right|^{2} d x+\frac{1}{2} \int_{\Gamma_{R}} \mathcal{I}_{h}\left(\kappa\left(\phi_{h}^{(m)}\right)^{2}\right) d s \\
+\sum_{j=1}^{m} \Delta t_{j}\left[\gamma\left\|\vec{u}_{h}^{(j)}\right\|_{D G}^{2}+\int_{\Omega} \sum_{i=1}^{N} D_{i} e^{\eta_{i, h}^{(j)}}\left|\nabla\left(\eta_{i, h}^{(j)}+q_{i} \phi_{h}^{(j)}\right)\right|^{2} d x\right] \\
\leq \int_{\Omega} \frac{\rho_{f}}{2}\left|\vec{u}_{h}^{(0)}\right|^{2}+\sum_{i=1}^{N} e^{\eta_{i, h}^{(0)}}\left(\eta_{i, h}^{(0)}-1\right)+\frac{\epsilon}{2}\left|\nabla \phi_{h}^{(0)}\right|^{2} d x+\frac{1}{2} \int_{\Gamma_{R}} \mathcal{I}_{h}\left(\kappa\left(\phi_{h}^{(0)}\right)^{2}\right) d s+C_{1},
\end{gathered}
$$

where $C_{1}$ depends on the number of charge carrying species, their initial masses, the electric permittivity and capacitance coefficients, but not on $T$. In the cases of no Dirichlet boundary conditions or homogeneous Dirichlet boundary conditions on $\phi_{h}$, the constant $C_{1}$ vanishes.

Proof. The proof of Theorem 4 closely follows that of Theorem 2, in addition to (36) for the NS variables. The only remaining terms are the cross terms between the PNP and NS systems, which cancel due to the strong divergence-free property of $\vec{u}_{h}^{(j)}$ and the continuity of the normal components across inter-element facets.

The conservation of mass follows from choosing $w_{h} \equiv 1 \in W_{h}$ in equation (38), as in Theorem 2. For an analogue of (20), follow the argument in the proof of Theorem 2 exactly to see

$$
\begin{aligned}
& \frac{\left[\mathcal{E}_{h}^{(j)}+\sum_{i=1}^{N} q_{i}\left(e^{\eta_{i, h}^{(j)}}, \phi_{h, D}\right)\right]-\left[\mathcal{E}_{h}^{(j-1)}+\sum_{i=1}^{N} q_{i}\left(e^{\eta_{i, h}^{(j-1)}}, \phi_{h, D}\right)\right]}{\Delta t_{j}} \\
\leq & -\sum_{i=1}^{N} \int_{\Omega} D_{i} e^{\eta_{i, h}^{(j)}}\left|\nabla\left(\eta_{i, h}^{(j)}+q_{i} \phi_{h}^{(j)}\right)\right|^{2} d x+\sum_{i=1}^{N}\left(e^{\eta_{i, h}^{(j)}} \vec{u}_{h}^{(j)}, \nabla\left(\eta_{i, h}^{(j)}+q_{i} \phi_{h}^{(j)}\right)\right) \\
= & -\sum_{i=1}^{N} \int_{\Omega} D_{i} e^{\eta_{i, h}^{(j)}}\left|\nabla\left(\eta_{i, h}^{(j)}+q_{i} \phi_{h}^{(j)}\right)\right|^{2} d x+\sum_{i=1}^{N}\left[\left(\vec{u}_{h}^{(j)}, \nabla e^{\eta_{i, h}^{(j)}}\right)+q_{i}\left(e^{\eta_{i, h}^{(j)}} \nabla \phi_{h}^{(j)}, \vec{u}_{h}^{(j)}\right)\right],
\end{aligned}
$$

where the discrete energy is recalled as

$$
\mathcal{E}_{h}^{(k)}=\int_{\Omega} \sum_{i=1}^{N} e^{\eta_{i, h}^{(k)}}\left(\eta_{i, h}^{(k)}-1\right)+\frac{\epsilon}{2}\left|\nabla \phi_{h}^{(k)}\right|^{2} d x+\frac{1}{2} \int_{\Gamma_{R}} \mathcal{I}_{h}\left(\kappa\left(\phi_{h}^{(k)}\right)^{2}\right) d s .
$$

Since $\vec{u}_{h}^{(j)}$ is strongly divergence free, has a continuous normal component across inter-element facets, and each $\eta_{i, h}^{(j)}$ is continuous,

$$
\sum_{i=1}^{N}\left(\vec{u}_{h}^{(j)}, \nabla e^{\eta_{i, h}^{(j)}}\right)=-\left(\nabla \cdot \vec{u}_{h}^{(j)}, \sum_{i=1}^{N} e^{\eta_{i, h}^{(j)}}\right)_{\mathcal{T}_{h}}+\sum_{\tau \in \mathcal{T}_{h}} \int_{\partial \tau}\left(\vec{u}_{h}^{(j)} \cdot \vec{n}_{\tau}\right) \sum_{i=1}^{N} e^{\eta_{i, h}^{(j)}} d s=0
$$


where the boundary conditions on $\vec{u}_{h}^{(j)}$ nullify all boundary terms.

Let $\zeta_{h}^{(j)}=\sum_{i=1}^{N} q_{i} e^{\eta_{i, h}^{(j)}} \in \mathcal{H}^{1}(\Omega)$ denote the total charge density. Combining (45) and (46) provides the bound

$$
\begin{aligned}
& \frac{\left[\mathcal{E}_{h}^{(j)}+\left(\zeta_{h}^{(j)}, \phi_{h, D}\right)\right]-\left[\mathcal{E}_{h}^{(j-1)}+\left(\zeta_{h}^{(j-1)}, \phi_{h, D}\right)\right]}{\Delta t_{j}} \\
& \quad \leq-\sum_{i=1}^{N} \int_{\Omega} D_{i} e^{\eta_{i, h}^{(j)}}\left|\nabla\left(\eta_{i, h}^{(j)}+q_{i} \phi_{h}^{(j)}\right)\right|^{2} d x+\left(\zeta_{h}^{(j)} \nabla \phi_{h}^{(j)}, \vec{u}_{h}^{(j)}\right) .
\end{aligned}
$$

For the NS terms, it follows from (35) that

$$
\begin{aligned}
D_{t, h}\left(\vec{u}_{h}^{(j)} ; \vec{u}_{h}^{(j)}, \vec{u}_{h}^{(j)}\right)-\frac{\rho_{f}}{\Delta t_{j}}\left(\vec{u}_{h}^{(j-1)}, \vec{u}_{h}^{(j)}\right)= & \frac{\rho_{f}}{\Delta t_{j}}\left(\vec{u}_{h}^{(j)}-\vec{u}_{h}^{(j-1)}, \vec{u}_{h}^{(j)}\right) \\
& +\frac{\rho_{f}}{2} \sum_{e \in \mathcal{F}_{h}} \int_{e}\left|\vec{u}_{h}^{(j)} \cdot \vec{n} \| \llbracket \llbracket\left(\vec{u}_{h}^{(j)}\right) \rrbracket\right|^{2} d s \\
\geq & \frac{\rho_{f}}{2 \Delta t_{j}}\left(\left\|\vec{u}_{h}^{(j)}\right\|_{0}^{2}-\left\|\vec{u}_{h}^{(j-1)}\right\|_{0}^{2}\right)
\end{aligned}
$$

and, choosing $q_{h}=p_{h}^{(j)}$ in (40),

$$
B_{h}\left(\vec{u}_{h}^{(j)}, p_{h}^{(j)}\right)=0
$$

Setting $\vec{v}_{h}=\vec{u}_{h}^{(j)}$ in (39) and employing the bounds (36), (48)-(49) gives

$$
\frac{\rho_{f}}{2 \Delta t_{j}}\left(\left\|\vec{u}_{h}^{(j)}\right\|_{0}^{2}-\left\|\vec{u}_{h}^{(j-1)}\right\|_{0}^{2}\right)+\gamma\left\|\vec{u}_{h}^{(j)}\right\|_{D G}^{2} \leq-\left(\zeta_{h}^{(j)} \nabla \phi_{h}^{(j)}, \vec{u}_{h}^{(j)}\right) .
$$

Adding (47) and (50) gives

$$
\begin{gathered}
\frac{\left[\frac{\rho_{f}}{2}\left\|\vec{u}_{h}^{(j)}\right\|_{0}^{2}+\mathcal{E}_{h}^{(j)}+\left(\zeta_{h}^{(j)}, \phi_{h, D}\right)\right]-\left[\frac{\rho_{f}}{2}\left\|\vec{u}_{h}^{(j-1)}\right\|_{0}^{2}+\mathcal{E}_{h}^{(j-1)}+\left(\zeta_{h}^{(j-1)}, \phi_{h, D}\right)\right]}{\Delta t_{j}} \\
\leq-\gamma\left\|\vec{u}_{h}^{(j)}\right\|_{D G}^{2}-\sum_{i=1}^{N} \int_{\Omega} D_{i} e^{\eta_{i, h}^{(j)}}\left|\nabla\left(\eta_{i, h}^{(j)}+q_{i} \phi_{h}^{(j)}\right)\right|^{2} d x . \quad(51)
\end{gathered}
$$

Summing over $j$, the bound (21), and Lemma 1 gives the desired energy estimate.

\section{Numerical experiments}

This section presents some numerical experiments that verify the viability, efficiency, and accuracy of computed solutions defined by the proposed discretization in the sections above. According to the discretizations in $\S \S 2-3$, 
a system of nonlinear elliptic equations must be solved at each time step. Recall that two commonly used techniques for resolving the nonlinear behavior are fixed point iteration (often referred to as Gummel iteration in the semiconductor literature) and Newton methods. Fixed point iteration is a very important tool in analysis, as convergence can be verified for more general problems; however, as a practical matter, it is often difficult to establish the rate of convergence to the nonlinear solution for this approach, motivating a quasi-Newton method for the experiments presented below. In these experiments, the computed Newton iterates approach the nonlinear finite element solution at a super-linear (nearly quadratic) rate. Due to the numerous computational difficulties present in solving the PNP equations, a significant amount of detail should be given to describe the nonlinear solver, the linearization procedure, upwinding schemes to preserve numerical stability of the linearized equations, and describing the linear solver for the arising linear algebraic systems. This lengthy discussion of the numerical solver is deferred to an upcoming publication [16], so that only the necessary and most basic aspects of the numerical solver are described here. The emphasis of these numerical results is that numerical schemes, when appropriately defined, compute approximate solutions to the PNP system that satisfy the energy estimates described above.

It is important to mention that the linearized equations resulting from a Newton-type approach lead to systems of linearized pdes that are potentially convection-dominated. This leads to potential algorithmic difficulties in preserving stability for the computed solution; so, some form of upwinding must be implemented to ensure accuracy. The well-studied edge-averaged finite element (EAFE) method is proven to provide stable numerical solutions that do not display spurious oscillatory behavior $[23,44]$. A point of emphasis here is that the nonlinear solution is stable, as verified by the energy estimates above, though the sequence of Newton iterates, defined by solving a sequence of linearized equations, is not necessarily stable.

A solver was implemented in $\mathrm{C}++$ that leverages some existing functionality of the FEniCS 1.3.0 [25] software package for generating systems of linear algebraic equations corresponding to an elliptic pde. Here, the elliptic pdes are the linearized pdes coming from Newton's method, with an EAFE approximation to improve numerical stability. Once the systems of algebraic equations are constructed, the Fast Auxilary Space Preconditioners (FASP) software package [7] is used to efficiently solve the resulting systems. The linear solvers in this software package use iterative techniques to efficiently provide approximate solutions, where convergence is defined by reducing the relative residual by a factor of $10^{-4}$ in all experiments. This tolerance is somewhat relaxed since each iterate solves an approximation of the Jacobian matrix, as this is a quasi-Newton scheme.

The first experiment presented here is designed to establish the convergence for the PNP solver at steady state; this solver is then used in a second numerical experiment to verify the discrete energy law (20) for the finite element formulation of the PNP system. Since the discrete solution is defined using a method-of-lines approach, this experiment also establishes the convergence rate 
of the computed solution to the true solution of the nonlinear elliptic equation at each time step. Several PNP systems are solved, where the permittivity coefficient, $\epsilon$, is tested for decreasing values. Testing the solver for small values of $\epsilon$ is important for many practical problems concerning semiconductors and biological applications, where this coefficient may be on the order of $10^{-4}$ to $10^{-8}$ after the system has been non-dimensionalized. For this experiment, the equation

$$
\begin{aligned}
-\nabla \cdot(\epsilon \nabla \phi) & =e^{\eta_{1}}-e^{\eta_{2}}+f_{0}, \\
\frac{\partial}{\partial t} e^{\eta_{1}} & =\nabla \cdot\left(e^{\eta_{1}} \nabla\left(\eta_{1}+\nabla \phi\right)\right)+f_{1}, \\
\frac{\partial}{\partial t} e^{\eta_{2}} & =\nabla \cdot\left(e^{\eta_{2}} \nabla\left(\eta_{2}-\nabla \phi\right)\right)+f_{2},
\end{aligned}
$$

is solved on the domain $\Omega=[-1,1] \times\left[-\frac{1}{2}, \frac{1}{2}\right] \times\left[-\frac{1}{2}, \frac{1}{2}\right]$, where $f_{0}, f_{1}, f_{2}$ are chosen so that the solution, for $x=\left(x_{1}, x_{2}, x_{3}\right) \in \Omega$, is

$\eta_{1}(x)=\frac{\log 10}{2}\left(x_{1}-1\right), \quad \eta_{2}(x)=-\frac{\log 10}{2}\left(x_{1}+1\right), \quad$ and $\quad \phi(x)=-\frac{2 \sinh \left(x_{1}\right)}{e-e^{-1}}$.

As this experiment is designed to test the numerical convergence to the nonlinear solution at steady state, Dirichlet boundary conditions are imposed at the ends of the domain to be consistent with the analytic expressions above, where $\left(\eta_{1}, \eta_{2}, \phi\right)=(-\log 10,0,1)$ when $x_{1}=-1$ and $\left(\eta_{1}, \eta_{2}, \phi\right)=(0,-\log 10,-1)$ when $x_{1}=1$. The iteration count for convergence to the nonlinear solutions (determined by reducing the relative residual by a factor of $10^{-10}$ ) are reported in Table 1, along with the $\mathcal{H}^{1}$ semi-norm of the error, given by

$$
\mathcal{H}^{1} \text { semi-norm }=\left(\left|e^{\eta_{1}}-e^{\eta_{1, h}}\right|_{1}^{2}+\left|e^{\eta_{2}}-e^{\eta_{2, h}}\right|_{1}^{2}+\left|\phi-\phi_{h}\right|_{1}^{2}\right)^{1 / 2} .
$$

It is clear that the Newton iterates converge in a reasonable number of iterations (fewer than 10 in all cases), which is encouraging for small values of $\epsilon$. Additionally, the convergence rate of the Newton iterates to the finite element solution is observed to be nearly quadratic, and the convergence of the finite element solution to the analytic solution is observed to be linear for all values of $\epsilon$ in Figure 1 with respect to the mesh size.

Note that the analytic solution in this experiment is independent of the permittivity, $\epsilon$, so that no boundary layers develop as $\epsilon$ decreases. This is due to the fact that the source terms, $f_{0}, f_{1}, f_{2}$, depend on $\epsilon$ to ensure the solution does not change. It is noted, however, that the stability results presented above are independent of the mesh resolution (but not shape regularity), so that the energy norm is bounded, which guarantees a bound on $\left|\phi_{h}\right|_{1}$ as long as the differential equation is solved to sufficient accuracy. This bound implies that $\phi$ is only permitted to oscillate up to some bounding quantity determined by the initial data and coefficients of the differential equation.

A second experiment validates that the energy estimate is satisfied. While this property is certainly true for the theoretical finite element solution satisfying (9)-(10), it is important to verify that the numerical solution, computed by 


\begin{tabular}{|c||c|c||c|c||c|c||c|c|}
\hline \multicolumn{1}{|c||}{} & \multicolumn{2}{|c|}{$20 \times 10 \times 10$} & \multicolumn{2}{|c|}{$40 \times 20 \times 20$} & \multicolumn{2}{|c|}{$60 \times 30 \times 30$} & \multicolumn{2}{|c|}{$80 \times 40 \times 40$} \\
\hline \hline 1 & 7 & $2.65 \times 10^{-3}$ & 6 & $6.67 \times 10^{-4}$ & 6 & $2.97 \times 10^{-4}$ & 6 & $1.67 \times 10^{-4}$ \\
$10^{-2}$ & 6 & $3.81 \times 10^{-3}$ & 6 & $9.80 \times 10^{-4}$ & 5 & $4.38 \times 10^{-4}$ & 5 & $2.47 \times 10^{-4}$ \\
$10^{-4}$ & 5 & $7.03 \times 10^{-3}$ & 5 & $2.37 \times 10^{-3}$ & 5 & $1.20 \times 10^{-3}$ & 5 & $7.18 \times 10^{-4}$ \\
$10^{-8}$ & 5 & $7.25 \times 10^{-3}$ & 9 & $2.61 \times 10^{-3}$ & 9 & $1.43 \times 10^{-3}$ & 9 & $9.34 \times 10^{-4}$ \\
\hline
\end{tabular}

Table 1: The count of Newton iterates to decrease the initial residual by a factor of $10^{-10}$ and the $\mathcal{H}^{1}$ semi-norm of the error of the computed solution once convergence is established.

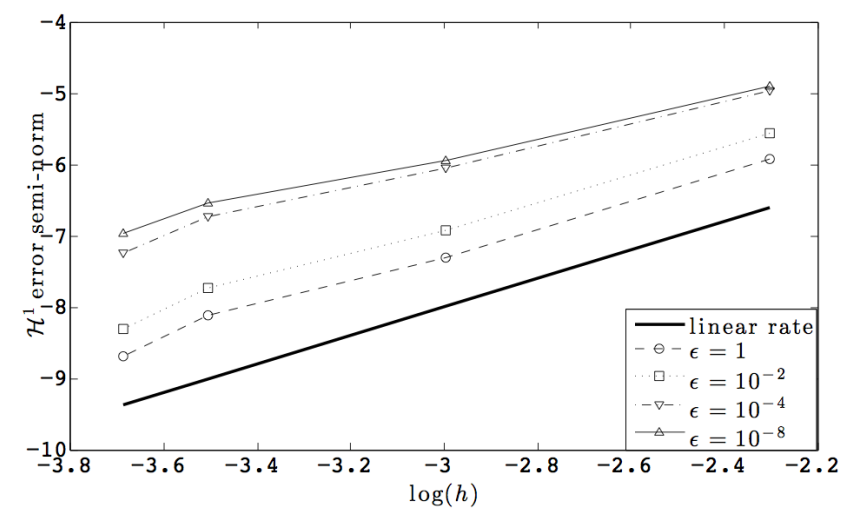

Figure 1: The logarithm of the error measured in the $\mathcal{H}^{1}$ semi-norm, plotted against the logarithm of the element diameter. The lines depict the log of the error for various values of $\epsilon$, where the thick line is a reference for linear convergence. 
inexact iterative methods, preserves this property. For this experiment, the spatial domain is $\Omega=[-1,1] \times\left[-\frac{1}{10}, \frac{1}{10}\right] \times\left[-\frac{1}{10}, \frac{1}{10}\right]$ and discretized by a $300 \times$ $10 \times 10$ mesh, and uniform time steps are taken with $\Delta t=\frac{1}{3000}$. The length of the time domain is determined by how well the solution from the previous time step solves the problem at the current time step. In particular, once the computed solution from the previous time step satisfies the residual of the current time step within a tolerance of $10^{-8}$, the system is considered to be in equilibrium; this occurs at $T=0.138$.

For this problem, the system defined by (1)-(3) is solved, with $N=2$, $\mu_{1}=\mu_{2}=D_{1}=D_{2}=1, q_{1}=1, q_{2}=-1$, and $\epsilon=\frac{1}{100}$. No-flux boundary conditions imposed on the Nernst-Planck equations and mixed homogeneous Dirichlet and inhomogeneous Neumann boundary conditions on the electrostatic potential:

$$
\begin{array}{rlrl}
\phi & =0, & \text { for } x_{1} & = \pm 1, \\
\epsilon \nabla \phi \cdot \vec{n} & =1, & \text { for } x_{1} \leq 0 \text { and } x_{3}=\frac{1}{10}, & \text { or } x_{1} \geq 0 \text { and } x_{3}=-\frac{1}{10}, \\
\epsilon \nabla \phi \cdot \vec{n} & =-1, \quad \text { for } x_{1} \leq 0 \text { and } x_{3}=-\frac{1}{10}, & \text { or } x_{1} \geq 0 \text { and } x_{3}=\frac{1}{10}, \\
\epsilon \nabla \phi \cdot \vec{n} & =0, \quad \text { for } x_{2}= \pm \frac{1}{10} .
\end{array}
$$

These boundary conditions verify that the constant $C_{1}=0$ in Theorem 2 and model surface charges, of alternating charge, lining opposite sides of a channel along the $x_{1}$ direction and electrode contacts at the ends of the channel. The experiment demonstrates for each time step that the discrete energy estimate,

$\delta E^{(j)}=\frac{\mathcal{E}_{h}^{(j)}-\mathcal{E}_{h}^{(j-1)}}{\Delta t} \leq-\int e^{\eta_{1, h}}\left|\nabla\left(\eta_{1, h}+\phi_{h}\right)\right|^{2}+e^{\eta_{2, h}}\left|\nabla\left(\eta_{2, h}-\phi_{h}\right)\right|^{2} d x \equiv-\Delta^{(j)}$,

is satisfied until convergence. To clearly illustrate that the energy estimate is satisfied, Figure 2 plots the quantity $\log \left(-\delta E^{(j)}\right)-\log \left(\Delta^{(j)}\right)$ over the time domain, where it is easy to see that this quantity is positive when the energy estimate is satisfied.

Numerical experiments for the electrokinetic system are still in development due to many considerations regarding the solver and DG discretization of the Navier-Stokes subsystem. Consequently, they are not included here and will be presented in an upcoming publication [16].

\section{Summary and concluding remarks}

In this paper, the energetic stability is established for the finite element solution to the PNP equations using a logarithmic transformation of the charge carrier densities. This energy estimate resembles the physical energy law that governs the PNP system in the continuous case, where the logarithmic transformation is an essential component for exactly evaluating the functionals describing the energy and rate of energy dissipation. This stability of the nonlinear 


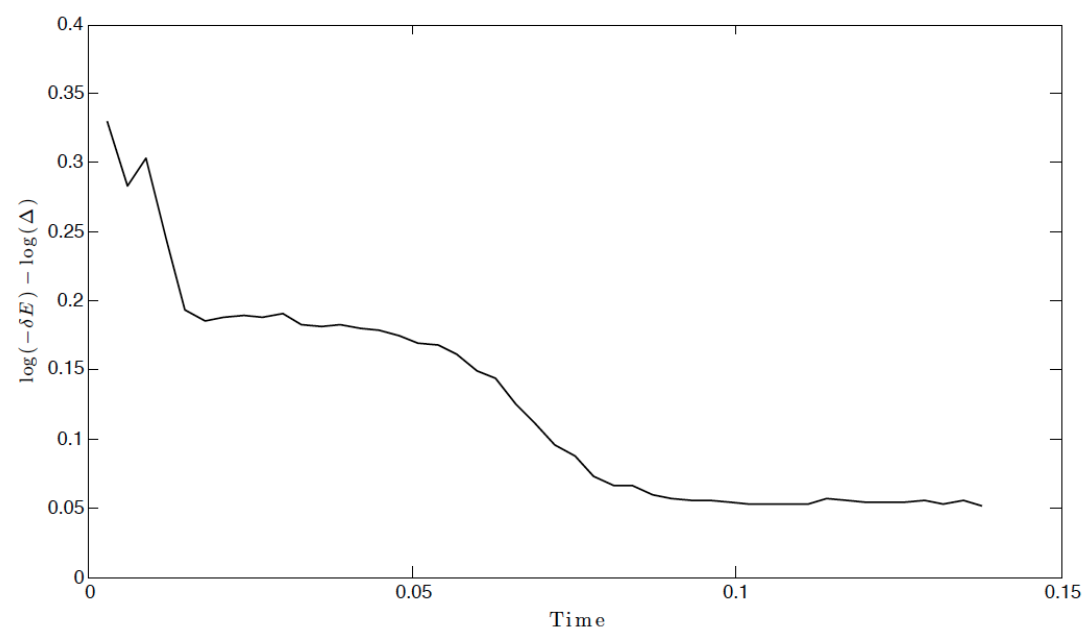

Figure 2: The difference, $\log (-\delta E)-\log (\Delta)$, plotted over the time domain until convergence.

finite element solution is shown to be independent of the time step size and the spatial resolution of the mesh. Furthermore, by imposing some additional constraints on the finite element mesh, a weak discrete maximum principle holds for the Poisson equation. This leads to an important extension of the energy estimate that covers the case of inhomogeneous boundary conditions imposed upon the electrostatic potential, which is highly relevant to many applications of the PNP system.

A second energy estimate is established for the finite element solution to the electrokinetic model that couples the PNP system with the incompressible NS equations. In addition to the logarithmic transformation, a key ingredient to establishing the stability for the electrostatic terms is the divergence-free property of the discrete solution to the NS equations, where this property can be achieved by using a div-conforming DG discretization of the NS equations. As seen in the proof of Theorem 4, the divergence-free property of the discrete fluid velocity naturally leads to the exact cancellation of the coupling terms between the PNP and NS equations.

In the previous section, some numerical experiments were carried out to demonstrate two properties of a computed solution. The first experiment numerically verified that the quasi-Newton scheme, using EAFE upwinding for the linearized equations, yields convergence to the finite element solution of the nonlinear pde in a reasonable number of iterations. Secondly, the computed solution using a quasi-Newton scheme is also shown to satisfy the energy law for the PNP system. The numerical solver for the PNP equations (with and without coupling to the NS equations) will be described in an upcoming publication [16].

While finite element solutions to the PNP and PNP-NS systems are shown 
to satisfy energy estimates, it is a matter of future work to prove an error estimate for these finite element solutions. This is not a matter of standard error analysis due to the nonlinearities of these problems, as well as the non-standard definition of the weak form, where the time derivative is not expressed in the $\mathcal{L}_{2}$ inner-product. In addition to the error estimate for the finite element solutions, one must provide some justification for the well-posedness and convergence of the computed sequence of Newton iterates to the finite element solution of the nonlinear pde. These two results, together with the energy estimates proved above, will verify that the proposed finite element discretization is indeed convergent.

Acknowledgement. The authors would like to extend their gratitude to Bob Eisenberg, who spent his time generously to explain various details of the PNP model and many of its applications while he visited Penn State. Also, the authors thank Xiaozhe $\mathrm{Hu}$ for relevant discussions regarding the discretization of the PNP model and contributions to the software used to run the numerical experiments. Finally, the authors thank Penn State's Center for Computational Mathematics and Applications for the group's help and contributions to various aspects of developing this work.

\section{References}

[1] B. Ayuso de Dios, F. Brezzi, L. D. Marini, J. Xu, And L. ZikATANOV, A simple preconditioner for a discontinuous Galerkin method for the Stokes problem, Journal of Scientific Computing, 58 (2014), pp. 517-547.

[2] R. E. Bank, W. Coughran JR, And L. C. Cowsar, The finite volume Scharfetter-Gummel method for steady convection diffusion equations, Computing and Visualization in Science, 1 (1998), pp. 123-136.

[3] R. E. BAnk, D. J. Rose, And W. Fichtner, Numerical methods for semiconductor device simulation, Electron Devices, IEEE Transactions on, 30 (1983), pp. 1031-1041.

[4] P. BILER AND J. DolBEAult, Long time behavior of solutions to Nernst-Planck and Debye-Hückel drift-diffusion systems, in Annales Henri Poincaré, vol. 1, Springer, 2000, pp. 461-472.

[5] P. Biler, W. Hebisch, and T. Nadzieja, The Debye system: existence and large time behavior of solutions, Nonlinear Analysis: Theory, Methods \& Applications, 23 (1994), pp. 1189-1209.

[6] J. F. Burgler, R. E. Bank, W. Fichtner, And R. K. Smith, A new discretization scheme for the semiconductor current continuity equations, Computer-Aided Design of Integrated Circuits and Systems, IEEE Transactions on, 8 (1989), pp. 479-489. 
[7] Center for Computational Mathematics and Applications, FASP: fast auxiliary space preconditioning. Mathematics Department, Pennsylvania State University, fasp.sourceforge.net/, 2015. Version 1.6.8.

[8] B. Cockburn, G. Kanschat, and D. Schötzau, A locally conservative LDG method for the incompressible Navier-Stokes equations, Mathematics of Computation, 74 (2005), pp. 1067-1095.

[9] S. DatTa, Electronic transport in mesoscopic systems, Cambridge university press, 1997.

[10] B. Eisenberg, Y. Hyon, And C. LiU, Energy variational analysis of ions in water and channels: Field theory for primitive models of complex ionic fluids, The Journal of chemical physics, 133 (2010), p. 104104.

[11] D. ERICKSOn AND D. LI, Influence of surface heterogeneity on electrokinetically driven microfluidic mixing, Langmuir, 18 (2002), pp. 1883-1892.

[12] M. Fortin And F. Brezzi, Mixed and hybrid finite element methods, New York: Springer-Verlag, 1991.

[13] T.-L. Horng, T.-C. Lin, C. Liu, And B. Eisenberg, Pnp equations with steric effects: a model of ion flow through channels, The Journal of Physical Chemistry B, 116 (2012), pp. 11422-11441.

[14] T.-L. Horng, T.-C. Lin, C. Liu, and R. Eisenberg, PNP equations with steric effects: a model of ion flow through channels, The Journal of Physical Chemistry B, 116 (2012), pp. 11422-11441.

[15] C.-Y. Hsieh, Y. Hyon, H. Lee, T.-C. Lin, And C. Liu, Transport of charged particles: entropy production and maximum dissipation principle, Journal of computational chemistry, 34 (2013), pp. 2065-2078.

[16] X. Hu, M. Metti, And J. Xu, Fast solvers for drift-diffusion and electrokinetic systems, in preparation, (2015).

[17] Y. Hyon, B. Eisenberg, and C. Liu, A mathematical model for the hard sphere repulsion in ionic solutions, Commun. Math. Sci, 9 (2011), pp. $459-475$.

[18] Y. HYon, D. Y. KWAK, AND C. LIU, Energetic variational approach in complex fluids: maximum dissipation principle, DCDS-A, 24 (2010), pp. 1291-1304.

[19] J. W. Jerome, Consistency of semiconductor modeling: an existence/stability analysis for the stationary van Roosbroeck system, SIAM journal on applied mathematics, 45 (1985), pp. 565-590.

[20] _ Analysis of charge transport: a mathematical study of semiconductor devices, Springer, Berlin, 1996. 
[21] R. Karnik, C. Duan, K. Castelino, H. Daiguji, and A. Majumdar, Rectification of ionic current in a nanofluidic diode, Nano letters, 7 (2007), pp. $547-551$.

[22] T. Kerkhoven And J. W. Jerome, $l^{\infty}$ stability of finite element approximations to elliptic gradient equations, Numerische Mathematik, 57 (1990), pp. $561-575$.

[23] R. D. Lazarov and L. T. Zikatanov, An exponential fitting scheme for general convection-diffusion equations on tetrahedral meshes, arXiv preprint arXiv:1211.0869, (2012).

[24] D. LI, Electrokinetics in microfluidics, vol. 2, Academic Press, 2004.

[25] A. Logg, K. A. Mardal, and G. Wells, Automated solution of differential equations by the finite element method: the FEniCS book, vol. 84, Springer Science \& Business Media, 2012.

[26] B. Lu, M. J. Holst, J. A. McCammon, and Y. Zhou, Poisson-NernstPlanck equations for simulating biomolecular diffusion-reaction processes I: finite element solutions, Journal of computational physics, 229 (2010), pp. 6979-6994.

[27] P. A. MARKOWICH, The stationary semiconductor device equations, Springer-Verlag New York, Inc., 1986.

[28] P. A. Markowich, C. A. Ringhofer, and C. Schmeiser, Semiconductor equations, Springer-Verlag New York, Inc., 1990.

[29] J. C. Maxwell, A dynamical theory of the electromagnetic field, Philosophical Transactions of the Royal Society of London, 155 (1865), p. $459 ? 512$.

[30] D. Meng, B. Zheng, G. Lin, And M. L. Sushko, Numerical solution of $3 D$ Poisson-Nernst-Planck Equations coupled with classical density functional theory for modeling ion and electron transport in a confined environment, Comm. Comp. Phys., 16 (2014), pp. 1298-1322.

[31] M. S. Mock, Analysis of mathematical models of semiconductor devices, vol. 3, Boole Press, 1983.

[32] Nernst, WALther, Die elektromotorische wirksamkeit der ionen, Z. Physik. Chem., 4 (1889).

[33] M. PlanCK, Über die erregung von electricität und wärme in electrolyten, Ann. Phys. Chem., 39 (1980).

[34] A. Prohl and M. Schmuck, Convergent discretizations for the NernstPlanck-Poisson system, Numerische Mathematik, 111 (2009), pp. 591-630. 
[35] — Convergent finite element discretizations of the Navier-StokesNernst-Planck-Poisson system, ESAIM: Mathematical Modelling and Numerical Analysis, 44 (2010), pp. 531-571.

[36] R. J. RYнам, Existence, uniqueness, regularity and long-term behavior for dissipative systems modeling electrohydrodynamics, arXiv preprint arXiv:0910.4973, (2009).

[37] R. SAcco And M. Stynes, Finite element methods for convectiondiffusion problems using exponential splines on triangles, Computers \& Mathematics with Applications, 35 (1998), pp. 35-45.

[38] D. Scharfetter And H. Gummel, Large signal analysis of a silicon Read diode oscillator, IEEE Trans. Electron Devices ED, 16 (1969), pp. 64-77.

[39] A. H. Schatz, A weak discrete maximum principle and stability of the finite element method in $l_{\infty}$ on plane polygonal domains. $i$, Mathematics of Computation, 34 (1980), pp. 77-91.

[40] S. ShIn, I. KANG, AND Y. Cho, Mixing enhancement by using electrokinetic instability under time-periodic electric field, Journal of Micromechanics and Microengineering, 15 (2005), p. 455.

[41] J. W. Slotboom, Computer-aided two-dimensional analysis of bipolar transistors, Electron Devices, IEEE Transactions on, 20 (1973), pp. 669679 .

[42] B. Tu, M. Chen, Y. Xie, L. Zhang, B. Eisenberg, and B. Lu, A parallel finite element simulator for ion transport through three-dimensional ion channel systems, Journal of computational chemistry, 34 (2013), pp. 2065-2078.

[43] J. Wu, V. SRinivasan, J. Xu, And C. WANG, Newton-Krylov-Multigrid algorithms for battery simulation, Journal of the electrochemical society, 149 (2002), pp. A1342-A1348.

[44] J. XU AND L. ZIKatanov, A monotone finite element scheme for convection-diffusion equations, Mathematics of Computation of the American Mathematical Society, 68 (1999), pp. 1429-1446.

[45] S. Xu, M. Chen, S. Majd, X. Yue, and C. Liu, Modeling and simulating asymmetrical conductance changes in gramicidin pores, Molecular Based Mathematical Biology, 2 (2014), pp. 34-55. 